\title{
A Degree Theory for Compact Perturbations of Monotone Type Operators and Application to Nonlinear Parabolic Problem
}

\author{
Teffera M. Asfaw \\ Department of Mathematics, Virginia Polytechnic Institute and State University, Blacksburg, VA 24061, USA \\ Correspondence should be addressed to Teffera M. Asfaw; tefferam@yahoo.com
}

Received 19 April 2017; Accepted 20 July 2017; Published 12 September 2017

Academic Editor: Stanislaw Migorski

Copyright (C) 2017 Teffera M. Asfaw. This is an open access article distributed under the Creative Commons Attribution License, which permits unrestricted use, distribution, and reproduction in any medium, provided the original work is properly cited.

\begin{abstract}
Let $X$ be a real locally uniformly convex reflexive Banach space with locally uniformly convex dual space $X^{*}$. Let $T: X \supseteq D(T) \rightarrow$ $2^{X^{*}}$ be maximal monotone, $S: X \rightarrow 2^{X^{*}}$ be bounded and of type $\left(S_{+}\right)$, and $C: D(C) \rightarrow X^{*}$ be compact with $D(T) \subseteq D(C)$ such that $C$ lies in $\Gamma_{\sigma}^{\tau}$ (i.e., there exist $\sigma \geq 0$ and $\tau \geq 0$ such that $\|C x\| \leq \tau\|x\|+\sigma$ for all $x \in D(C)$ ). A new topological degree theory is developed for operators of the type $T+S+C$. The theory is essential because no degree theory and/or existence result is available to address solvability of operator inclusions involving operators of the type $T+S+C$, where $C$ is not defined everywhere. Consequently, new existence theorems are provided. The existence theorem due to Asfaw and Kartsatos is improved. The theory is applied to prove existence of weak solution (s) for a nonlinear parabolic problem in appropriate Sobolev spaces.
\end{abstract}

\section{Introduction: Preliminaries}

In what follows, the norm of the spaces $X$ and $X^{*}$ will be denoted by $\|\cdot\|$. For $x \in X$ and $x^{*} \in X^{*}$, the pairing $\left\langle x^{*}, x\right\rangle$ denotes the value $x^{*}(x)$. Let $X$ and $Y$ be real Banach spaces. For an operator $T: X \rightarrow 2^{Y}$, we define the domain $D(T)$ of $T$ by $D(T)=\{x \in X: T x \neq \emptyset\}$, and the range $R(T)$ of $T$ by $R(T)=\bigcup_{x \in D(T)} T x$. We also use the symbol $G(T)$ for the graph of $T: G(T)=\left\{\left(x, x^{*}\right): x \in D(T), x^{*} \in T x\right\}$. An operator $T: X \supset D(T) \rightarrow Y$ is "demicontinuous" if it is continuous from the strong topology of $D(T)$ to the weak topology of $Y$. It is "compact" if it is strongly continuous and maps bounded subsets of $D(T)$ to relatively compact subsets of $Y$. An operator $T: X \supset D(T) \rightarrow 2^{Y}$ is "bounded" if it maps each bounded subset of $D(T)$ into a bounded subset of $Y$. It is "finitely continuous" if it is upper semicontinuous from each finite dimensional subspace $F$ of $X$ to the weak topology of $Y$. Let $\phi:[0, \infty) \rightarrow(-\infty, \infty)$ be a continuous and strictly increasing function such that $\phi(t) \rightarrow \infty$ as $t \rightarrow \infty$. The mapping $J_{\phi}: X \rightarrow 2^{X^{*}}$ defined by

$$
\begin{aligned}
& J_{\phi}(x) \\
& =\left\{x^{*} \in X^{*}:\left\langle x^{*}, x\right\rangle=\phi(\|x\|)\|x\|,\left\|x^{*}\right\|=\phi(\|x\|)\right\}
\end{aligned}
$$

is called the "duality mapping" associated with $\phi$. As a consequence of the Hahn-Banach theorem, it is well-known that $J_{\phi}(x) \neq \emptyset$ for all $x \in X$. Since $X$ and $X^{*}$ are locally uniformly convex, $J_{\phi}$ is single valued, bounded, monotone, and bicontinuous. The following definitions are needed throughout the paper.

Definition 1. An operator $T: X \supset D(T) \rightarrow 2^{X^{*}}$ is said to be

(i) "monotone" if for every $x \in D(T), y \in D(T), u^{*} \in T x$, and $v^{*} \in T y$, we have $\left\langle u^{*}-v^{*}, x-y\right\rangle \geq 0$;

(ii) "maximal monotone" if $T$ is monotone and $R(T+$ $\lambda J)=X^{*}$ for every $\lambda>0$; that is, $T$ is maximal monotone if and only if $T$ is monotone and $\left\langle u^{*}-\right.$ $\left.u_{0}^{*}, x-x_{0}\right\rangle \geq 0$ for every $\left(x, u^{*}\right) \in G(T)$ implies $x_{0} \in D(T)$ and $u_{0}^{*} \in T x_{0}$;

(iii) "coercive" if either $D(T)$ is bounded or there exists a function $\psi:[0, \infty) \rightarrow(-\infty, \infty)$ such that $\psi(t) \rightarrow \infty$ as $t \rightarrow \infty$ and $\left\langle y^{*}, x\right\rangle \geq \psi(\|x\|)\|x\|$ for all $x \in D(T)$ and $y^{*} \in T x$;

(iv) "weakly coercive" if either $D(T)$ is bounded or $|T x| \rightarrow$ $\infty$ as $\|x\| \rightarrow \infty$, where for each $x \in D(T),|T x|=$ $\inf \left\{\left\|v^{*}\right\|: v^{*} \in T x\right\}$. 
It is important to note here that the class of weakly coercive operators includes the classes of coercive operators. For a maximal monotone operator $T: X \supseteq D(T) \rightarrow 2^{X^{*}}$, we know that $R(T+\lambda J)=X^{*}$ for all $\lambda>0$ and $(T+\lambda J)^{-1}: X^{*} \rightarrow$ $D(T)$ is single valued and demicontinuous. In addition, the operator $T_{t}: X \rightarrow X^{*}, t \in(0, \infty)$, defined by $T_{t} x=\left(T^{-1}+\right.$ $\left.t J^{-1}\right)^{-1} x$, is the "Yosida approximant" of $T$. It is bounded, continuous, and maximal monotone with domain $X$ such that $T_{t} x \rightarrow T^{(0)} x$ as $t \rightarrow 0^{+}$, for every $x \in D(T)$, where $\left\|T^{(0)} x\right\|=$ $\inf \left\{\left\|y^{*}\right\|: y^{*} \in T x\right\}$. Furthermore, the operator $J_{t}: X \rightarrow$ $D(T)$, defined by $J_{t} x=x-t J^{-1}\left(T_{t} x\right)$, is called the "Yosida resolvent" of $T$. It is continuous, $T_{t} x \in T\left(J_{t} x\right)$ for every $x \in X$, and $\lim _{t \rightarrow 0} J_{t} x=x$ for all $x \in \overline{\operatorname{co} D(T)}$, where $\operatorname{coD}(T)$ is the convex hull of the set $D(T)$. Furthermore, for each $x \in D(T)$, $\left\|T_{t} x\right\| \leq|T x|$ for all $t>0$. Browder and Hess [1] introduced the following definitions. The original definition of single valued pseudomonotone operator is due to Brèzis [2].

Definition 2. An operator $T: X \supset D(T) \rightarrow 2^{X^{*}}$ is said to be

(a) "pseudomonotone" if the following conditions are satisfied:

(i) For every $x \in D(T), T x$ is nonempty, closed, convex, and bounded subset of $X^{*}$;

(ii) $T$ is finitely continuous; that is, for every $x_{0} \in$ $D(T) \cap F$ and every weak neighborhood $V$ of $T x_{0}$ in $X^{*}$, there exists a neighborhood $U$ of $x_{0}$ in $F$ such that $T U \subset V$;

(iii) for each sequence $\left\{x_{n}\right\} \subset D(T)$ with $y_{n}^{*} \in T x_{n}$ such that $x_{n} \rightarrow x_{0} \in D(T)$ and

$$
\limsup _{n \rightarrow \infty}\left\langle y_{n}^{*}, x_{n}-x_{0}\right\rangle \leq 0 \text {, }
$$

we have that, for every $x \in D(T)$, there exists $y^{*}(x) \in T x_{0}$ such that

$$
\left\langle y^{*}(x), x_{0}-x\right\rangle \leq \liminf _{n \rightarrow \infty}\left\langle y_{n}^{*}, x_{n}-x\right\rangle ;
$$

in particular, letting $x_{0}$ in place of $x$ in the above inequality, the pseudomonotonicity of $T$ implies

$$
\liminf _{n \rightarrow \infty}\left\langle y_{n}^{*}, x_{n}-x_{0}\right\rangle \geq 0
$$

(b) "of type $\left(S_{+}\right)$" if (i) and (ii) of (a) hold and for each sequence $\left\{x_{n}\right\}$ in $D(T)$ such that $x_{n} \rightarrow x_{0}$ in $X$ as $n \rightarrow$ $\infty$ and every $w_{n}^{*} \in S x_{n}$ with

$$
\limsup _{n \rightarrow \infty}\left\langle w_{n}^{*}, x_{n}-x_{0}\right\rangle \leq 0
$$

we have $x_{n} \rightarrow x_{0} \in D(T)$ and there exists a subsequence of $\left\{w_{n}^{*}\right\}$, denoted again by $\left\{w_{n}^{*}\right\}$, such that $w_{n}^{*} \rightarrow w_{0}^{*} \in T x_{0}$ as $n \rightarrow \infty$

(c) "of type (S)" if (i) and (ii) of (a) hold and for any sequence $x_{n} \in D(T), v_{n}^{*} \in T x_{n}$ such that $v_{n}^{*} \rightarrow v_{0}^{*}$ as $n \rightarrow \infty$, it follows that there exists a subsequence of $\left\{x_{n}\right\}$, denoted again by $\left\{x_{n}\right\}$, such that $x_{n} \rightarrow x_{0}$ as $n \rightarrow \infty$.
It is not difficult to see that the class of operators of type $(S)$ includes the classes of operators of type $\left(S_{+}\right)$. Furthermore, it holds that $T+C$ is of type $(S)$ provided that $T$ is of type $(S)$ and $C$ is compact. The main goals of this paper are

(i) to develop suitable degree theory for operators of the type $T+S+C$, where $T: X \supseteq D(T) \rightarrow 2^{X^{*}}$ is maximal monotone, $S: X \rightarrow 2^{X^{*}}$ is bounded of type $\left(S_{+}\right)$, and $C: D(C) \rightarrow X^{*}$ is compact with $D(T) \subseteq D(C)$ and sublinear; that is, there exist $\tau \geq 0$ and $\sigma \geq 0$ such that $\|C x\| \leq \tau\|x\|+\sigma$ for all $x \in D(C)$. The existing degree theories for operators of the type $T+S$ cannot be used to treat inclusions involving operators of the type $T+S+C$ because the compact operator is not everywhere defined. For recent degree theories for multivalued bounded $\left(S_{+}\right)$or bounded pseudomonotone perturbations of arbitrary maximal monotone operators, the reader is referred to the papers by Asfaw and Kartsatos [3], Asfaw [4], Adhikari and Kartsatos [5], and the references therein. In these theories, the maximal monotone operator is arbitrary and $\left(S_{+}\right)$and/or pseudomonotone operator is everywhere defined. The original degree mapping due to Browder [6] is for operators of the type $T+f$, where $f$ is single valued bounded operator of type $\left(S_{+}\right)$defined from the closure of a nonempty, bounded, and open subset $G$ of $X$. Hu and Papageorgiou [7] generalized Browder's theory for multivalued compact perturbation of $T+f$, where the compact operator is defined on $\bar{G}$. All these theories do not include the case where $C$ is not defined on $\bar{G}$, in particular, when $D(C)$ contains $D(T)$. In view of these, our work in developing a degree theory for operators of the type $T+S+C$, where $C$ is a compact operator with $D(T) \subseteq D(C)$, is essential. It is worth mentioning that the theory associated with (i) is a generalization of the previous degree theories for bounded $\left(S_{+}\right)$perturbations of maximal monotone operators due to Browder [6], Kobayashi and Otani [8], $\mathrm{Hu}$ and Papageorgiou [7], Asfaw and Kartsatos [3], and the references therein. The most general degree theory currently available which is due to Asfaw [9] is for pseudomonotone perturbations of the sum of two maximal monotone operators with one of the maximal monotone operators which is of type $\Gamma_{\phi}^{\beta}$;

(ii) to derive existence theorem(s) in order to establish solvability of operator inclusion problems involving operators of the type $T+S+C$. Consequently, the theory developed in (i) is applied to prove existence of solution for the inclusion problem $f^{*} \epsilon$ $\overline{(T+S+C)\left(D(T) \cap B_{R}(0)\right)}$ provided that there exists $R=R\left(f^{*}\right)>0$ such that

$$
\left\langle v^{*}+w^{*}+C x-f^{*}, x\right\rangle>0
$$

for all $x \in D(T) \cap \partial B_{R}(0), v^{*} \in T x$, and $w^{*} \in S x$; that is, $\overline{R(T+S+C)}=X^{*}$ provided that $T+S+C$ is coercive. The result is a generalization of the existence result due to Asfaw and Kartsatos [3, Theorem 17] for 
the operator $T+S$. This result yields the surjectivity of $T+S+C$ provided that $T+S+C$ is coercive and either $S$ is bounded of type $\left(S_{+}\right)$or $T+S$ is operator of type $(S)$.

Throughout the paper, we shall use the following definition of a homotopy of class $\left(S_{+}\right)$.

Definition 3. Let $t \in[0,1]$ and $S^{t}: X \supset D\left(S^{t}\right) \rightarrow 2^{X^{*}}$. The family $\left\{S^{t}\right\}_{t \in[0,1]}$ is said to be a "homotopy of type $\left(S_{+}\right)$" if the following are true:

(i) For each $t \in[0,1], x \in D\left(S^{t}\right), S^{t} x$ is a nonempty, closed, convex, and bounded subset of $X^{*}$.

(ii) For each $t \in[0,1], S^{t}$ is finitely continuous.

(iii) Let $\left\{t_{n}\right\} \subset[0,1], x_{n} \in D\left(S^{t_{n}}\right)$ be such that $t_{n} \rightarrow t_{0}$ and $x_{n} \rightarrow x_{0} \in X$. Let $f_{n} \in S^{t_{n}} x_{n}$ be such that

$$
\limsup _{n \rightarrow \infty}\left\langle f_{n}, x_{n}-x_{0}\right\rangle \leq 0 \text {. }
$$

Then $x_{n} \rightarrow x_{0} \in D\left(S^{t_{0}}\right)$ and there exists a subsequence of $\left\{f_{n}\right\}$, denoted again by $\left\{f_{n}\right\}$, such that $f_{n} \rightarrow f \in S^{t_{0}} x_{0}$ as $n \rightarrow \infty$. [10].

The following lemma is due to Ibrahimou and Kartsatos

Lemma 4. Let $T: X \supset D(T) \rightarrow 2^{X^{*}}$ be maximal monotone and $G \subset X$ be bounded. Let $0<s_{1} \leq s_{2}, 0<t_{1}<t_{2}$. Let $T^{s}:=s T$. Then there exists a constant $K_{1}>0$, independent of $t$ and $s$, such that $\left\|T_{t}^{s} u\right\| \leq K_{1}$ for all $u \in \bar{G}, s \in\left[s_{1}, s_{2}\right]$, and $t \in\left[t_{1}, t_{2}\right]$.

For basic definitions and further properties of mappings of monotone type, the reader is referred to Barbu [11], Pascali and Sburlan [12], Browder and Hess [1], and Zeidler [13].

The content of the following important lemma is due to Brezis et al. [14].

Lemma 5. Let $B$ be a maximal monotone set in $X \times X^{*}$. If $\left(u_{n}, u_{n}^{*}\right) \in B$ such that $u_{n} \rightarrow u$ in $X, u_{n}^{*} \rightarrow u^{*}$ in $X^{*}$, and

$$
\limsup _{n \rightarrow \infty}\left\langle u_{n}^{*}-u^{*}, u_{n}-u\right\rangle \leq 0,
$$

then $\left(u, u^{*}\right) \in B$ and $\left\langle u_{n}^{*}, u_{n}\right\rangle \rightarrow\left\langle u^{*}, u\right\rangle$ as $n \rightarrow \infty$.

Browder [6] introduced the concept of a pseudomonotone homotopy as given below.

Definition 6. Let $\left\{T^{t}\right\}_{t \in[0,1]}$ be a family of maximal monotone operators from $X$ to $2^{X^{*}}$ such that $0 \in T^{t}(0), t \in[0,1]$. Then $\left\{T^{t}\right\}_{t \in[0,1]}$ is called a "pseudomonotone homotopy" if it satisfies the following equivalent conditions:

(i) Suppose that $t_{n} \rightarrow t_{0} \in[0,1]$ and $\left(x_{n}, y_{n}\right) \in G\left(T^{t_{n}}\right)$ are such that $x_{n} \rightarrow x_{0}$ in $X, y_{n} \rightarrow y_{0}$ in $X^{*}$ and

$$
\limsup _{n \rightarrow \infty}\left\langle y_{n}, x_{n}\right\rangle \leq\left\langle y_{0}, x_{0}\right\rangle \text {. }
$$

Then $\left(x_{0}, y_{0}\right) \in G\left(T^{t_{0}}\right)$ and $\lim _{n \rightarrow \infty}\left\langle y_{n}, x_{n}\right\rangle=$ $\left\langle y_{0}, x_{0}\right\rangle$. (ii) The mapping $\phi: X^{*} \times[0,1] \rightarrow X$ defined by

$$
\phi(w, t):=\left(T^{t}+J\right)^{-1}(w)
$$

is continuous.

(iii) For each $w \in X^{*}$, the mapping $\phi_{w}:[0,1] \rightarrow X$ defined by

$$
\phi_{w}(t):=\left(T^{t}+J\right)^{-1}(w)
$$

is continuous.

(iv) For any $(x, y) \in G\left(T^{t_{0}}\right)$ and any sequence $t_{n} \rightarrow t_{0}$, there exists a sequence $\left(x_{n}, y_{n}\right) \in G\left(T^{t_{n}}\right)$ such that $x_{n} \rightarrow x$ and $y_{n} \rightarrow y$ as $n \rightarrow \infty$.

For a maximal monotone operator $T: X \supseteq$ $D(T) \rightarrow 2^{X^{*}}$, Kobayashi and Otani [8] proved that the family $\{t T\}_{t \in[0,1]}$ is a pseudomonotone homotopy of maximal monotone operators if and only if $T$ is densely defined. It is worth mentioning that the proof of this fact does not require the hypothesis $0 \in T(0)$. It is essential herein to mention that the original degree theory for singlevalue $\left(S_{+}\right)$perturbations of maximal monotone operators is due to Browder [6]. For a generalization of Browder's degree for multivalued compact perturbations of $T+f$, where $T: X \supset D(T) \rightarrow 2^{X^{*}}$ is maximal monotone and $f:$ $\bar{G} \rightarrow X^{*}$ is bounded demicontinuous of type $\left(S_{+}\right)$, the reader is referred to the paper due to $\mathrm{Hu}$ and Papageorgiou [7]. For existence results for compact perturbation of maximal monotone operators, the reader is referred to the paper due to Kartsatos [15]. For a relevant degree mapping for single multivalued operator of type $\left(S_{+}\right)$, we cite the paper of Zhang and Chen [16]. Recent developments on degree theories for perturbations of the sum of two maximal monotone operators can be found in the papers due to Adhikari and Kartsatos [5] and Asfaw [4].

In Section 2 we construct a degree mapping for operators of the type $T+S+C$, where $T: X \supseteq D(T) \rightarrow 2^{X^{*}}$ is maximal monotone, $S: X \rightarrow 2^{X^{*}}$ is bounded and of type $\left(S_{+}\right)$or bounded pseudomonotone, and $C: D(C) \rightarrow X^{*}$ is compact with $D(T) \subseteq D(C)$ and satisfies a sublinearity condition. The existence of solutions for operator inclusion problems of the type $\mathrm{Tu}+\mathrm{Su}+\mathrm{Cu} \ni f^{*}$ is included in Section 3. In Section 4, the theory is applied to establish existence of weak solution(s) for a nonlinear parabolic problem in appropriate Sobolve spaces.

\section{Degree Theory for $T+S+C$ with $D(T) \subseteq D(C)$}

2.1. Degree Theory for $T+S+C$ with $S$ Bounded and of Type $\left(S_{+}\right)$. The goal of this section is to develop a degree theory for operators of the type $T+S+C$, where $T: X \supset D(T) \rightarrow 2^{X^{*}}$ is maximal monotone, $S: X \rightarrow 2^{X^{*}}$ is bounded and of type $\left(S_{+}\right)$, and $C: D(C) \rightarrow X^{*}$ is compact with $D(T) \subseteq D(C)$. Throughout the paper, we assume that $C$ belongs to $\Gamma_{\sigma}^{\tau}$ (i.e., there exist $\sigma \geq 0$ and $\tau \geq 0$ such that $\|C x\| \leq \tau\|x\|+\sigma$ for all $x \in D(C)$ ). To this end, we start by proving the following useful lemma. 
Lemma 7. Let $G$ be a nonempty, bounded, and open subset of $X$. Let $T: X \supseteq D(T) \rightarrow 2^{X^{*}}$ be maximal monotone, $S: X \rightarrow$ $2^{X^{*}}$ be bounded and of type $\left(S_{+}\right)$, and $C: X \supseteq D(C) \rightarrow X^{*}$ be compact with $D(T) \subseteq D(C)$ such that $C$ belongs to class $\Gamma_{\sigma}^{\tau}$. Assume, further, that $f^{*} \notin(T+S+C)(D(T) \cap \partial G)$. Then there exists $\varepsilon_{0}>0$ such that $d\left(T_{\varepsilon}+S+C J_{\varepsilon}, G, f^{*}\right)$ is well-defined and independent of $\varepsilon \in\left(0, \varepsilon_{0}\right]$.

Proof. In the first step, we claim that there exists $\varepsilon_{0}>0$ such that $d\left(T_{\varepsilon}+S+C J_{\varepsilon}, G, f^{*}\right)$ is well-defined for all $\varepsilon \in\left(0, \varepsilon_{0}\right]$. Suppose that this is false; that is, there exist $\varepsilon_{n} \downarrow 0^{+}, x_{n} \in \partial G$, and $w_{n}^{*} \in S x_{n}$ such that

$$
v_{n}^{*}+w_{n}^{*}+C J_{\varepsilon_{n}} x_{n}=f^{*} \quad \forall n
$$

where $v_{n}^{*}=T_{\varepsilon_{n}} x_{n}$. By the definitions of $T_{\varepsilon_{n}}$ and $J_{\varepsilon_{n}}$, we have

$$
\begin{aligned}
& J_{\varepsilon_{n}} x_{n}=x_{n}-\varepsilon_{n} J^{-1}\left(v_{n}^{*}\right) \in D(T), \\
& v_{n}^{*} \in T\left(J_{\varepsilon_{n}} x_{n}\right) \forall n .
\end{aligned}
$$

Since $\left\{x_{n}\right\}$ and $S$ are bounded, it follows that $\left\{w_{n}^{*}\right\}$ is bounded. Since $C$ belongs to $\Gamma_{\sigma}^{\tau}$, we get that

$$
\begin{aligned}
\left\|v_{n}^{*}\right\| & \leq\left\|f^{*}-w_{n}^{*}\right\|+\left\|C J_{\varepsilon_{n}} x_{n}\right\| \leq \kappa_{0}+\tau\left\|J_{\varepsilon_{n}} x_{n}\right\|+\sigma \\
& =\kappa_{0}+\tau\left\|x_{n}-\varepsilon_{n} J^{-1}\left(v_{n}^{*}\right)\right\|+\sigma \\
& \leq \kappa_{0}+\tau\left\|x_{n}\right\|+\tau \varepsilon_{n}\left\|v_{n}^{*}\right\|+\sigma
\end{aligned}
$$

for all $n$, where $\kappa_{0}$ is an upper bound for $\left\{f^{*}-w_{n}^{*}\right\}$. This yields the estimate

$$
\left(1-\tau \varepsilon_{n}\right)\left\|v_{n}^{*}\right\| \leq \kappa_{0}+\tau\left\|x_{n}\right\|+\sigma
$$

for all $n$. Since $\varepsilon_{n} \downarrow 0^{+}$and $\left\{x_{n}\right\}$ is bounded, it follows that $\left\{v_{n}^{*}\right\}$ and $\left\{J_{\varepsilon_{n}} x_{n}\right\}$ are bounded. The compactness of $C$ implies the boundedness of $\left\{C J_{\varepsilon_{n}} x_{n}\right\}$. Now, assume without loss of generality that $x_{n} \rightarrow x_{0}, v_{n}^{*} \rightarrow v_{0}^{*}$, and $w_{n}^{*} \rightarrow w_{0}^{*}$ as $n \rightarrow \infty$. Since $C$ is compact, we may assume, by passing into a subsequence if necessary, that $C J_{\varepsilon_{n}} x_{n} \rightarrow g_{0}^{*}$ as $n \rightarrow \infty$. The maximality of $T$ along with Lemma 5 gives

$$
\liminf _{n \rightarrow \infty}\left\langle v_{n}^{*}, x_{n}-x_{0}\right\rangle \geq 0
$$

that is, we obtain from (12) that

$$
\begin{aligned}
\limsup _{n \rightarrow \infty}\left\langle w_{n}^{*}, x_{n}-x_{0}\right\rangle \\
=\limsup _{n \rightarrow \infty}\left(-\left\langle v_{n}^{*}+C J_{\varepsilon_{n}} x_{n}-f^{*}, x_{n}-x_{0}\right\rangle\right) \\
=-\liminf _{n \rightarrow \infty}\left(\left\langle v_{n}^{*}+C J_{\varepsilon_{n}} x_{n}-f^{*}, x_{n}-x_{0}\right\rangle\right) \\
\leq-\liminf _{n \rightarrow \infty}\left\langle v_{n}^{*}, x_{n}-x_{0}\right\rangle \\
\quad-\liminf _{n \rightarrow \infty}\left\langle C J_{\varepsilon_{n}} x_{n}-f^{*}, x_{n}-x_{0}\right\rangle \\
=-\liminf _{n \rightarrow \infty}\left\langle v_{n}^{*}, x_{n}-x_{0}\right\rangle \leq 0 .
\end{aligned}
$$

Since $S$ is of type $\left(S_{+}\right)$, we conclude that $x_{n} \rightarrow x_{0} \in \partial G$ as $n \rightarrow \infty$ and $w_{0}^{*} \in S x_{0}$. Consequently, using (12) we arrive at

$$
\limsup _{n \rightarrow \infty}\left\langle v_{n}^{*}, x_{n}-x_{0}\right\rangle \leq 0 \text {. }
$$

The maximality of $T$ along with Lemma 5 yields $x_{0} \in D(T) \cap$ $\partial G$ and $v_{0}^{*} \in T x_{0}$ and $\left\langle v_{n}^{*}, x_{n}\right\rangle \rightarrow\left\langle v_{0}^{*}, x_{0}\right\rangle$ as $n \rightarrow \infty$. Since $C$ is compact and $J_{\varepsilon_{n}} x_{n}=x_{n}-\varepsilon_{n} J^{-1}\left(v_{n}^{*}\right) \rightarrow x_{0} \in D(T) \subseteq D(C)$ as $n \rightarrow \infty$, it follows that $C J_{\varepsilon_{n}} x_{n} \rightarrow C x_{0}=g_{0}^{*}$ as $n \rightarrow \infty$. Letting $n \rightarrow \infty$ in (12), we get $f^{*} \in(T+S+C)(D(T) \cap \partial G)$. However, this is impossible. Thus, there exists $\varepsilon_{0}>0$ such that $d\left(T_{\varepsilon}+S+C J_{\varepsilon}, G, f^{*}\right)$ is well-defined for all $\varepsilon \in\left(0, \varepsilon_{0}\right]$.

Next, we shall prove that $d\left(T_{\varepsilon}+S+C J_{\varepsilon}, G, f^{*}\right)$ is independent of $\varepsilon \in\left(0, \varepsilon_{0}\right]$. Let $\varepsilon_{i} \in\left(0, \varepsilon_{0}\right](i=1,2)$ be such that $0<\varepsilon_{1}<\varepsilon_{2} \leq \varepsilon_{0}, q(t)=t \varepsilon_{1}+(1-t) \varepsilon_{2}, t \in[0,1]$. We consider the homotopy operator

$$
H(t, x)=T_{q(t)} x+S x+C J_{q(t)} x, \quad(t, x) \in[0,1] \times \bar{G} .
$$

We will show that the family $\{H(t, \cdot)\}_{t \in[0,1]}$ is a homotopy of class $\left(S_{+}\right)$such that $0 \notin H(t, \partial G)$ for all $t \in[0,1]$. To this end, let $x_{n} \in \bar{G}, w_{n}^{*} \in S x_{n}, t_{n} \in[0,1], f_{n}^{*}=T_{q\left(t_{n}\right)} x_{n}+w_{n}^{*}+C J_{q\left(t_{n}\right)} x_{n}$, $x_{n} \rightarrow x_{0}$, and $t_{n} \rightarrow t_{0}$ as $n \rightarrow \infty$ be such that

$$
\limsup _{n \rightarrow \infty}\left\langle f_{n}^{*}, x_{n}-x_{0}\right\rangle \leq 0 \text {. }
$$

Since $\left\{x_{n}\right\}$ and $S$ are bounded, it follows that $\left\{w_{n}^{*}\right\}$ is also bounded. Since $q\left(t_{n}\right) \in\left[\varepsilon_{1}, \varepsilon_{2}\right]$ for all $n$, we apply Lemma 4 to conclude that $\left\{T_{q\left(t_{n}\right)} x_{n}\right\}$ and $\left\{J_{q\left(t_{n}\right)} x_{n}\right\}$ are bounded. On the other hand, we see that

$$
\begin{aligned}
& \limsup _{n \rightarrow \infty}\left\langle w_{n}^{*}, x_{n}-x_{0}\right\rangle \leq \limsup _{n \rightarrow \infty}\left\langle f_{n}^{*}\right. \\
& \left.\quad-\left(T_{q\left(t_{n}\right)} x_{n}-T_{q\left(t_{n}\right)} x_{0}+C J_{q\left(t_{n}\right)} x_{n}\right), x_{n}-x_{0}\right\rangle \\
& \quad-\lim _{n \rightarrow \infty}\left\langle T_{q\left(t_{n}\right)} x_{0}, x_{n}-x_{0}\right\rangle .
\end{aligned}
$$

By the compactness of $C$, we may assume without loss of generality that $C J_{q\left(t_{n}\right)} x_{n} \rightarrow h_{0}^{*}$ as $n \rightarrow \infty$. Since $q\left(t_{n}\right) \rightarrow q=$ $q\left(t_{0}\right)>0$ as $n \rightarrow \infty$, we use the continuity of $(0, \infty) \times X \ni$ $(t, x) \rightarrow T_{t}(x)$ ([3], Lemma 6) to conclude that $T_{q\left(t_{n}\right)} x_{0} \rightarrow$ $T_{q} x_{0}$ as $n \rightarrow \infty$. Combining these along with the monotonicity of $T_{q\left(t_{n}\right)}$, we obtain

$$
\limsup _{n \rightarrow \infty}\left\langle w_{n}^{*}, x_{n}-x_{0}\right\rangle \leq 0 .
$$

Since $S$ is of type $\left(S_{+}\right)$, we see that $x_{n} \rightarrow x_{0}$ as $n \rightarrow \infty$ and there exists a subsequence of $\left\{w_{n}^{*}\right\}$, denoted again by $\left\{w_{n}^{*}\right\}$, such that $w_{n}^{*} \rightarrow w_{0}^{*} \in S x_{0}$ as $n \rightarrow \infty$. Since $(0, \infty) \times X \ni$ $(t, x) \mapsto T_{t} x$ is continuous, we conclude that

$$
\begin{array}{r}
f_{n}^{*} \rightarrow T_{q} x_{0}+w_{0}^{*}+C J_{q} x_{0} \in T_{q} x_{0}+S x_{0}+C J_{q} x_{0} \\
\text { as } n \longrightarrow \infty ;
\end{array}
$$

that is, $\{H(t, \cdot)\}_{t \in[0,1]}$ is a homotopy of class $\left(S_{+}\right)$such that $0 \notin H(t, \partial G)$ for all $t \in[0,1]$. Therefore, $d\left(H(t, \cdot), G, f^{*}\right)$ is independent of $t \in[0,1]$; that is, $d\left(T_{\varepsilon_{1}}+S+C J_{\varepsilon_{1}}, G, f^{*}\right)=$ $d\left(T_{\varepsilon_{2}}+S+C J_{\varepsilon_{2}}, G, f^{*}\right)$. Since $\varepsilon_{1}$ and $\varepsilon_{2}$ are arbitrary in $\left(0, \varepsilon_{0}\right]$, we conclude that $d\left(T_{\varepsilon}+S+C J_{\varepsilon}, G, f^{*}\right)$ is well-defined and independent of $\varepsilon \in\left(0, \varepsilon_{0}\right]$. This completes the proof. 
Based on Lemma 7, the associated degree mapping is defined as follows.

Definition 8. Let $G$ be a nonempty, bounded, and open subset of $X, T: X \supset D(T) \rightarrow 2^{X^{*}}$ be maximal monotone, $S: X \rightarrow$ $2^{X^{*}}$ be bounded and of type $\left(S_{+}\right)$, and $C: X \supseteq D(C) \rightarrow X^{*}$ be compact with $D(T) \subseteq D(C)$ and belonging to the class $\Gamma_{\sigma}^{\tau}$. Assume, further, that $f^{*} \notin(T+S+C)(D(T) \cap \partial G)$. Then the degree mapping $d$ for $T+S+C$ at $f^{*} \in X^{*}$ with respect to $G$ is defined by

$$
d\left(T+S+C, G, f^{*}\right)=\lim _{\varepsilon \downarrow 0^{+}} d_{S_{+}}\left(T_{\varepsilon}+S+C J_{\varepsilon}, G, f^{*}\right)
$$

where $d_{S_{+}}$is the degree mapping for multivalued bounded operators of type $\left(S_{+}\right)$from [16].

\subsection{Basic Properties of the Degree}

Theorem 9. Let $G$ be a nonempty, bounded, and open subset of $X$. Let $T: X \supset D(T) \rightarrow 2^{X^{*}}$ be maximal monotone, $S:$ $X \rightarrow 2^{X^{*}}$ be bounded and of type $\left(S_{+}\right)$, and $C: D(C) \rightarrow X^{*}$ be compact with $D(T) \subseteq D(C)$ such that $C$ belongs to $\Gamma_{\sigma}^{\tau}$. Then the following properties hold:

(i) (Normalization) $d(J, G, 0)=1$ if $0 \in G$ and $d(J, G$, $0)=0$ if $0 \notin \bar{G}$.

(ii) (Existence) if $f^{*} \notin(T+S+C)(D(T) \cap \partial G)$ and $d(T+$ $\left.S+C, G, f^{*}\right) \neq 0$, then $f^{*} \in(T+S+C)(D(T) \cap G)$.

(iii) (Decomposition) let $G_{1}$ and $G_{2}$ be nonempty, disjoint, and open subsets of $G$ such that $f^{*} \notin(T+S+C)(D(T) \cap$ $\left.\left(\bar{G} \backslash\left(G_{1} \cup G_{2}\right)\right)\right)$. Then

$$
\begin{aligned}
d\left(T+S+C, G, f^{*}\right)= & d\left(T+S+C, G_{1}, f^{*}\right) \\
& +d\left(T+S+C, G_{2}, f^{*}\right) .
\end{aligned}
$$

(iv) (Translation invariance) let $f^{*} \notin(T+S+C)(D(T) \cap$ $\partial G)$. Then we have

$$
d\left(T+S+C-f^{*}, G, 0\right)=d\left(T+S+C, G, f^{*}\right) .
$$

(v) Let $M(t, x)=T x+t\left(S_{1} x+C x\right)+(1-t) S_{2} x,(t, x) \epsilon$ $[0,1] \times(D(T) \cap \bar{G})$, where $S_{i}: X \rightarrow 2^{X^{*}}(i=1,2)$ is bounded and of type $\left(S_{+}\right)$and $0 \notin M(t, D(T) \cap \partial G)$ for all $t \in[0,1]$. Then $d(M(t, \cdot), G, 0)$ is independent of $t \in[0,1]$.

(vi) Let $0 \in G, N(t, x)=t\left(T x+S_{1} x+C x\right)+(1-t) S_{2} x$, $(t, x) \in[0,1] \times(D(T) \cap \bar{G})$, where $T: X \supset D(T) \rightarrow X^{*}$ is densely defined maximal monotone and positively homogeneous of order $\alpha>0, S_{i}: X \rightarrow 2^{X^{*}}(i=1,2)$ is bounded and of type $\left(S_{+}\right)$such that $\left\langle u^{*}, x\right\rangle \geq\|x\|^{2}$ for all $x \in X, u^{*} \in S_{2} x$, and $0 \notin N(t, D(T) \cap \partial G)$ for all $t \in[0,1]$. Assume, further, that $0 \notin M(t, D(T) \cap \partial G)$ for all $t \in[0,1]$. Then $d(N(t, \cdot), G, 0)$ is independent of $t \in[0,1]$.
Proof. The proof of (i) follows by setting $T=\{0\}$ and $C=\{0\}$. To prove (ii), assume that $f^{*} \notin(T+S+C)(D(T) \cap \partial G)$ and $d\left(T+S+C, G, f^{*}\right) \neq 0$. By the definition of $d$, there exists $\varepsilon_{0}>0$ such that $d\left(T_{\varepsilon}+S+C J_{\varepsilon}, G, f^{*}\right) \neq 0$ for all $\varepsilon \in\left(0, \varepsilon_{0}\right]$; that is, for each $\varepsilon_{n} \downarrow 0^{+}$there exist $x_{n} \in D(T) \cap G$ and $w_{n}^{*} \in S x_{n}$ such that

$$
v_{n}^{*}+w_{n}^{*}+C J_{\varepsilon_{n}} x_{n}=f^{*}, \quad v_{n}^{*}=T_{\varepsilon_{n}} x_{n} \forall n .
$$

Since $S$ is bounded, it follows that $\left\{w_{n}^{*}\right\}$ is bounded. By using $\Gamma_{\sigma}^{\tau}$ condition on $C$ along with the arguments used in the proofs of Lemma 7, it is easy to see that $\left\{v_{n}^{*}\right\}$ and $\left\{J_{\varepsilon_{n}} x_{n}\right\}$ are bounded. Assume without loss of generality that $x_{n} \rightarrow x_{0}$, $w_{n}^{*} \rightarrow w_{0}^{*}, v_{n}^{*} \rightarrow v_{0}^{*}$, and $C J_{\varepsilon_{n}} x_{n} \rightarrow g_{0}^{*}$ as $n \rightarrow \infty$. By the maximality of $T$, the $\left(S_{+}\right)$condition on $S$, and the arguments used in the proof of Lemma 7, we conclude that $x_{0} \in D(T) \cap G$, $v_{0}^{*} \in T x_{0}$, and $w_{0}^{*} \in S x_{0}$ such that $v_{0}^{*}+w_{0}^{*}+C x_{0}=f^{*}$. This shows that $f^{*} \in(T+S+C)(D(T) \cap G)$.

Next we prove (iii). Suppose the hypotheses in (iii) hold. By the definition of $d$, we see that $d\left(T+S+C, G, f^{*}\right)=d\left(T_{\varepsilon}+\right.$ $\left.S+C J_{\varepsilon}, G, f^{*}\right)$ for all sufficiently small $\varepsilon>0$. Since $T_{\varepsilon}+S+C J_{\varepsilon}$ is bounded and of type $\left(S_{+}\right)$, the decomposition property of the degree mapping for multivalued $\left(S_{+}\right)$operators implies

$$
\begin{aligned}
d\left(T+S+C, G, f^{*}\right)= & \lim _{\varepsilon \downarrow 0^{+}} d\left(T_{\varepsilon}+S+C J_{\varepsilon}, G, f^{*}\right) \\
= & \lim _{\varepsilon \downarrow 0^{+}} d\left(T_{\varepsilon}+S+C J_{\varepsilon}, G_{1}, f^{*}\right) \\
& +\lim _{\varepsilon \downarrow 0^{+}} d\left(T_{\varepsilon}+S+C J_{\varepsilon}, G_{2}, f^{*}\right) \\
= & d\left(T+S+C, G_{1}, f^{*}\right) \\
& +d\left(T+S+C, G_{2}, f^{*}\right) ;
\end{aligned}
$$

that is, (iii) holds.

(iv) Suppose that $f^{*} \notin(T+S+C)(D(T) \cap \partial G)$; that is, $0 \notin\left(T+S+C-f^{*}\right)(D(T) \cap \partial G)$. This implies that $d(T+S+C-$ $\left.f^{*}, G, 0\right)$ is well-defined. Since $d\left(T+S+C-f^{*}, G, 0\right)=d\left(T_{\varepsilon}+\right.$ $\left.S+C J_{\varepsilon}-f^{*}, G, 0\right)$, by the translation property of the degree mapping for multivalued bounded operators of type $\left(S_{+}\right)$, we see that $d\left(T_{\varepsilon}+S+C J_{\varepsilon}-f^{*}, G, 0\right)=d\left(T_{\varepsilon}+S+C J_{\varepsilon}, G, f^{*}\right)$. Thus,

$$
\begin{aligned}
d( & \left.T+S+C-f^{*}, G, 0\right) \\
& =\lim _{\varepsilon \downarrow 0^{+}} d\left(T_{\varepsilon}+S+C J_{\varepsilon}-f^{*}, G, 0\right) \\
& =\lim _{\varepsilon \downarrow 0^{+}} d\left(T_{\varepsilon}+S+C J_{\varepsilon}, G, f^{*}\right) \\
& =d\left(T+S+C, G, f^{*}\right) .
\end{aligned}
$$

(v) Suppose that $0 \notin M(t, D(T) \cap \partial G)$ for all $t \in[0,1]$, where $M(t, x)=T x+t\left(S_{1} x+C x\right)+(1-t) S_{2} x,(t, x) \in[0,1] \times$ $(D(T) \cap \bar{G})$. For every $\varepsilon>0$, we consider

$$
\begin{aligned}
M_{\varepsilon}(t, x)=T_{\varepsilon} x+t\left(S_{1} x+C J_{\varepsilon} x\right)+ & (1-t) S_{2} x, \\
& (t, x) \in[0,1] \times \bar{G} .
\end{aligned}
$$


We shall show that there exists $\varepsilon_{0}>0$ such that $d\left(M_{\varepsilon}(t, \cdot)\right.$, $G, 0)$ is well-defined and independent of all $(t, \varepsilon) \in[0,1] \times$ $\left(0, \varepsilon_{0}\right]$. To do this, we assume to the contrary that there exist $\varepsilon_{n} \downarrow 0^{+}, x_{n} \in \partial G, t_{n} \in[0,1], w_{n}^{*} \in S_{1} x_{n}$, and $u_{n}^{*} \in S_{2} x_{n}$ such that

$$
\begin{aligned}
v_{n}^{*}+t_{n}\left(w_{n}^{*}+C J_{\varepsilon_{n}} x_{n}\right)+\left(1-t_{n}\right) u_{n}^{*}= & , \\
& v_{n}^{*}=T_{\varepsilon_{n}} x_{n} \forall n .
\end{aligned}
$$

Since $\left\{x_{n}\right\}, S_{1}$, and $S_{2}$ are bounded, it follows that $\left\{w_{n}^{*}\right\}$ and $\left\{u_{n}^{*}\right\}$ are bounded. By the $\Gamma_{\sigma}^{\tau}$ condition on $C$, the boundedness of $S_{1}$ and $S_{2}$, and the arguments used in the proof of Lemma 7, we conclude that $\left\{J_{\varepsilon_{n}} x_{n}\right\}$ and $\left\{v_{n}^{*}\right\}$ are bounded. Assume without loss of generality that $t_{n} \rightarrow t_{0}, x_{n} \rightarrow x_{0}, w_{n}^{*} \rightarrow w_{0}^{*}, u_{n}^{*} \rightarrow$ $u_{0}^{*}, v_{n}^{*} \rightarrow v_{0}^{*}, J_{\varepsilon_{n}} x_{n}=x_{n}-\varepsilon_{n} J^{-1}\left(v_{n}^{*}\right) \rightarrow x_{0}$, and $C J_{\varepsilon_{n}} x_{n} \rightarrow$ $g_{0}^{*}$ as $n \rightarrow \infty$. Suppose that $t_{0}=0$. We have $v_{n}^{*}+u_{n}^{*} \rightarrow 0$ as $n \rightarrow \infty$. Since $S_{2}$ is of type $\left(S_{+}\right)$, it follows that $x_{n} \rightarrow x_{0} \in \partial G$, $v_{0}^{*} \in D(T)$, and $0 \in T x_{0}+S_{2} x_{0}$; that is, $0 \in\left(T+S_{2}\right)(D(T) \cap \partial G)$, that is, $0 \in M(0, D(T) \cap \partial G)$. However, this is impossible. A similar proof covers the case $t_{0} \neq 1$. Assume $t_{0} \in(0,1)$. Suppose there exists a subsequence of $\left\{t_{n}\right\}$, denoted again by $\left\{t_{n}\right\}$, such that $t_{n} \geq \tau_{0}>0$ for all $n$. Since $T$ is maximal monotone, Lemma 5 implies $\liminf _{n \rightarrow \infty}\left\langle v_{n}^{*}, x_{n}-x_{0}\right\rangle \geq 0$. As a result, (31) implies

$$
\begin{aligned}
\limsup _{n \rightarrow \infty} & \left\langle w_{n}^{*}, x_{n}-x_{0}\right\rangle \\
= & -\liminf _{n \rightarrow \infty}\left(\frac{1}{t_{n}}\left\langle v_{n}^{*}+\left(1-t_{n}\right) u_{n}^{*}, x_{n}-x_{0}\right\rangle\right) \\
& -\limsup _{n \rightarrow \infty}\left\langle C J_{\varepsilon_{n}} x_{n}, x_{n}-x_{0}\right\rangle \\
= & -\frac{1}{t_{0}} \liminf _{n \rightarrow \infty}\left\langle v_{n}^{*}+\left(1-t_{n}\right) u_{n}^{*}, x_{n}-x_{0}\right\rangle \\
& -\liminf _{n \rightarrow \infty}\left\langle C J_{\varepsilon_{n}} x_{n}, x_{n}-x_{0}\right\rangle \leq 0 .
\end{aligned}
$$

Since $S_{1}$ is of type $\left(S_{+}\right)$, it follows that $x_{n} \rightarrow x_{0} \in \partial G$ as $n \rightarrow$ $\infty$ and $w_{0}^{*} \in S x_{0}$. Moreover, one can show that $x_{0} \in D(T)$, $v_{0}^{*} \in T x_{0}$, and $u_{0}^{*} \in S_{2} x_{0}$ so that $0=v_{0}^{*}+t_{0}\left(w_{0}^{*}+C x_{0}\right)+(1-$ $\left.t_{0}\right) u_{0}^{*}$; that is, $0 \in\left(T+t_{0}(S+C)\right)(D(T) \cap \partial G)$. However, this is a contradiction.

To show that $d\left(M_{\varepsilon}(t, \cdot), G, 0\right)$ is constant for all $t \in[0,1]$ and $\varepsilon \in\left(0, \varepsilon_{0}\right]$, with $\varepsilon_{0}$ as in the proof of (ii), we let $0<\varepsilon_{1}<$ $\varepsilon_{2} \leq \varepsilon_{0}$ and consider the homotopy operator

$$
\begin{aligned}
\widetilde{M}(t, x)= & T_{q(t)} x+t\left(S_{1} x+C J_{q(t)} x\right)+(1-t) S_{2} x, \\
& q(t)=t \varepsilon_{1}+(1-t) \varepsilon_{2}, \quad(t, x) \in[0,1] \times \bar{G} .
\end{aligned}
$$

Since for each $t \in[0,1] T_{q(t)}$ is monotone, $S_{1}$ and $S_{2}$ are bounded and of type $\left(S_{+}\right)$and $C J_{q(t)}$ is compact, it follows that $M_{1}(t, \cdot)$ is bounded demicontinuous and of type $\left(S_{+}\right)$. It is not hard to verify that $0 \notin \widetilde{M}(t, \partial G)$ for all $t \in[0,1]$. As in the arguments used in the proof of Lemma 7, we shall show that $\{\widetilde{M}(t, \cdot)\}_{t \in[0,1]}$ is a homotopy of class $\left(S_{+}\right)$. To this end, let $x_{n} \in \bar{G}$ and $t_{n} \in[0,1]$ be such that $x_{n} \rightarrow x_{0}$ and $t_{n} \rightarrow t_{0} \in[0,1]$ as $n \rightarrow \infty, w_{n}^{*} \in S_{1} x_{n}$ and $u_{n}^{*} \in S_{2} x_{n}$ be such that

$$
\begin{aligned}
& \limsup _{n \rightarrow \infty}\left\langle T_{q\left(t_{n}\right)} x_{n}+t_{n}\left(w_{n}^{*}+C J_{q\left(t_{n}\right)} x_{n}\right)\right. \\
& \left.\quad+\left(1-t_{n}\right) u_{n}^{*}, x_{n}-x_{0}\right\rangle \leq 0
\end{aligned}
$$

Since $T_{q\left(t_{n}\right)}$ is monotone with domain $X$, it follows that

$$
\left\langle T_{q\left(t_{n}\right)} x_{n}-T_{q\left(t_{n}\right)} x_{0}, x_{n}-x_{0}\right\rangle \geq 0
$$

for all $n$. Since $q\left(t_{n}\right) \rightarrow q\left(t_{0}\right)>0$ as $n \rightarrow \infty$ and $(0, \infty) \times$ $X \ni(t, x) \rightarrow T_{t} x$ is continuous, we get $T_{q\left(t_{n}\right)} x_{0} \rightarrow T_{q\left(t_{0}\right)} x_{0}$ as $n \rightarrow \infty$. As a result of this, we get

$$
\begin{gathered}
\limsup _{n \rightarrow \infty}\left\langle T_{q\left(t_{n}\right)} x_{0}+t_{n}\left(w_{n}^{*}+C J_{q\left(t_{n}\right)} x_{n}\right)\right. \\
\left.\quad+\left(1-t_{n}\right) u_{n}^{*}, x_{n}-x_{0}\right\rangle \leq 0
\end{gathered}
$$

that is,

$$
\begin{aligned}
& \limsup _{n \rightarrow \infty}\left\langle t_{n}\left(w_{n}^{*}+C J_{q\left(t_{n}\right)} x_{n}\right)+\left(1-t_{n}\right) u_{n}^{*}, x_{n}-x_{0}\right\rangle \\
& \quad \leq 0 .
\end{aligned}
$$

Since $q(t) \in\left[\varepsilon_{1}, \varepsilon_{2}\right]$ for all $t \in[0,1]$, an application of Lemma 4 says that there exists $\gamma_{0}>0$ independent of $n$ such that $\left\|T_{q\left(t_{n}\right)} x_{n}\right\| \leq \gamma_{0}$ for all $n$. In addition, by the definition of $J_{q\left(t_{n}\right)}$, we see that

$$
\begin{aligned}
\left\|J_{q\left(t_{n}\right)} x_{n}\right\| & =\left\|x_{n}-q\left(t_{n}\right) J^{-1}\left(T_{q\left(t_{n}\right)} x_{n}\right)\right\| \\
& \leq\left\|x_{n}\right\|+q\left(t_{n}\right)\left\|J^{-1}\left(T_{q\left(t_{n}\right)} x_{n}\right)\right\| \\
& =\left\|x_{n}\right\|+q\left(t_{n}\right)\left\|T_{q\left(t_{n}\right)} x_{n}\right\| \leq\left\|x_{n}\right\|+q\left(t_{n}\right) \gamma_{0}
\end{aligned}
$$

for all $n$. Since $\left\{x_{n}\right\}$ is bounded, the boundedness of $\left\{J_{q\left(t_{n}\right)} x_{n}\right\}$ follows. By the compactness of $C$, we may assume without loss of generality that $C J_{q\left(t_{n}\right)} x_{n} \rightarrow g_{0}^{*}$ as $n \rightarrow \infty$. As a result of this, we get

$$
\begin{aligned}
0 \geq & \limsup _{n \rightarrow \infty}\left\langle t_{n}\left(w_{n}^{*}+C J_{q\left(t_{n}\right)} x_{n}\right)+\left(1-t_{n}\right) u_{n}^{*}, x_{n}\right. \\
& \left.-x_{0}\right\rangle=\limsup _{n \rightarrow \infty}\left\langle t_{n} w_{n}^{*}+\left(1-t_{n}\right) u_{n}^{*}, x_{n}-x_{0}\right\rangle \\
& +\lim _{n \rightarrow \infty} t_{n}\left\langle C J_{q\left(t_{n}\right)} x_{n}, x_{n}-x_{0}\right\rangle=\limsup _{n \rightarrow \infty}\left\langle t_{n} w_{n}^{*}\right. \\
& \left.+\left(1-t_{n}\right) u_{n}^{*}, x_{n}-x_{0}\right\rangle .
\end{aligned}
$$

Let $t_{0} \in(0,1)$. The boundedness of $\left\{w_{n}^{*}\right\}$ and $\left\{u_{n}^{*}\right\}$ imply

$$
\limsup _{n \rightarrow \infty}\left\langle t_{0} w_{n}^{*}+\left(1-t_{0}\right) u_{n}^{*}, x_{n}-x_{0}\right\rangle \leq 0 .
$$

Since $S_{1}$ and $S_{2}$ are bounded and of type $\left(S_{+}\right)$, it follows that $x_{n} \rightarrow x_{0}$ and there exist subsequences of $\left\{w_{n}^{*}\right\}$ and $\left\{u_{n}^{*}\right\}$, denoted again by $\left\{w_{n}^{*}\right\}$ and $\left\{u_{n}^{*}\right\}$, respectively, such that $w_{n}^{*} \rightarrow$ $w_{0}^{*} \in S_{1} x_{0}$ and $u_{n}^{*} \rightarrow u_{0}^{*} \in S_{2} x_{0}$ as $n \rightarrow \infty$. Moreover, by 
the continuity of $(0, \infty) \times X \ni(t, x) \mapsto T_{t} x$, it follows that $T_{q\left(t_{n}\right)} x_{n} \rightarrow T_{q\left(t_{0}\right)} x_{0}$ as $n \rightarrow \infty$. From the continuity of $J$ and $C$, we obtain that

$$
\begin{aligned}
& J_{q\left(t_{n}\right)} x_{n}=x_{n}-q\left(t_{n}\right) J^{-1}\left(T_{q\left(t_{n}\right)} x_{n}\right) \longrightarrow \\
& x_{0}-q\left(t_{0}\right) J^{-1}\left(T_{q\left(t_{0}\right)} x_{0}\right)=y_{0} \in D(T) \subset D(C)
\end{aligned}
$$

as $n \rightarrow \infty$ and $C J_{q\left(t_{n}\right)} x_{n} \rightarrow C y_{0}=g_{0}^{*}$ as $n \rightarrow \infty$. Thus, we arrive at

$$
\begin{aligned}
T_{q\left(t_{n}\right)} x_{n} & =\frac{1}{q\left(t_{n}\right)} J\left(x_{n}-J_{q\left(t_{n}\right)} x_{n}\right) \longrightarrow \\
\frac{1}{q\left(t_{0}\right)} J\left(x_{0}-y_{0}\right) & =h_{0}^{*}
\end{aligned}
$$

as $n \rightarrow \infty$. Since $T_{q\left(t_{n}\right)} x_{n} \in T\left(J_{q\left(t_{n}\right)} x_{n}\right)$ for all $n$, by the maximality of $T$, we conclude that $y_{0} \in D(T) \subset D(C)$, $h_{0}^{*} \in T y_{0}$, and $\left\langle T_{q\left(t_{n}\right)} x_{n}, J_{q\left(t_{n}\right)} x_{n}\right\rangle \rightarrow\left\langle h_{0}^{*}, y_{0}\right\rangle$ as $n \rightarrow \infty$. Therefore, we get

$$
\begin{aligned}
& T_{q\left(t_{n}\right)} x_{n}+t_{n}\left(w_{n}^{*}+C J_{q\left(t_{n}\right)} x_{n}\right)+\left(1-t_{n}\right) u_{n}^{*} \\
& \rightarrow T_{q\left(t_{0}\right)} x_{0}+t_{0}\left(w_{0}^{*}+C y_{0}\right)+\left(1-t_{0}\right) u_{0}^{*}
\end{aligned}
$$

as $n \rightarrow \infty$. The proofs of the cases $t_{0}=0$ and $t_{0}=1$ can be completed in an analogous manner. The details are omitted here. Thus the family $\{\widetilde{M}(t, \cdot)\}_{t \in[0,1]}$ is a homotopy of class $\left(S_{+}\right)$; that is, $d(\widetilde{M}(t, \cdot), G, 0)$ is independent of $t \in[0,1]$. This implies

$$
\begin{aligned}
d(\widetilde{M}(t, \cdot) . G, 0) & =d(\widetilde{M}(1, \cdot), G, 0) \\
& =d\left(T_{\varepsilon_{1}}+S_{1}+C J_{\varepsilon_{1}}, G, 0\right) \\
& =d(\widetilde{M}(0, \cdot), G, 0) \\
& =d\left(T_{\varepsilon_{2}}+S_{2}, G, 0\right) \quad \forall t \in[0,1] .
\end{aligned}
$$

Consequently, by the definition of $d(M(t, \cdot), G, 0)$, there exists $\varepsilon_{0}>0$ such that

$$
\begin{aligned}
d( & M(t, \cdot), G, 0)=d\left(T+t S_{1}+(1-t) S_{2}+t C, G, 0\right) \\
& =d\left(T_{\varepsilon}+t S_{1}+(1-t) S_{2}+t C J_{\varepsilon}, G, 0\right) \\
& =d\left(T_{\varepsilon_{2}}+S_{2}, G, 0\right) \quad \forall t \in[0,1], \varepsilon \in\left(0, \varepsilon_{0}\right] .
\end{aligned}
$$

This proves that $d(M(t, \cdot), G, 0)$ is independent of $t \in[0,1]$; that is, the proof (iv) is complete.

(vi) Suppose the hypotheses in (vi) hold. Since, for each $t \in[0,1], t T$ is maximal monotone, let $T_{\varepsilon}^{t} x=(t T)_{\varepsilon} x, x \in X$, be Yosida approximant of $t T$ and $J_{\varepsilon}^{t}$ be the Yosida resolvent of $t T$. Since $t S_{1}+(1-t) S_{2}$ is bounded and of type $\left(S_{+}\right)$and $t C$ is compact with $D(t C)=D(C)$ for all $t \in[0,1]$, we shall show that there exists $\varepsilon_{0}>0$ such that $d\left(T_{\varepsilon}^{t}+t S_{1}+(1-t) S_{2}+\right.$ $\left.t C J_{\varepsilon}^{t}, G, 0\right)$ is independent of $t \in[0,1]$ and $\varepsilon \in\left(0, \varepsilon_{0}\right]$. Assume that this does not hold; that is, there exist $\varepsilon_{n} \downarrow 0^{+}, x_{n} \in D(T) \cap$ $\partial G, w_{n}^{*} \in S_{1} x_{n}, u_{n}^{*} \in S_{2} x_{n}$, and $t_{n} \in[0,1]$ such that

$$
v_{n}^{*}+t_{n} w_{n}^{*}+\left(1-t_{n}\right) u_{n}^{*}+t_{n} C y_{n}=0
$$

for all $n$, where $v_{n}^{*}=T_{\varepsilon_{n}}^{t_{n}} x_{n} \in t_{n} T\left(y_{n}\right)$ and $y_{n}=J_{\varepsilon_{n}}^{t_{n}} x_{n} \in D(T)$. By using the $\Gamma_{\sigma}^{\tau}$ condition on $C$, we arrive at

$$
\begin{aligned}
\left\|v_{n}^{*}\right\| & \leq t_{n}\left\|w_{n}^{*}\right\|+\left(1-t_{n}\right)\left\|u_{n}^{*}\right\|+t_{n}\left\|C y_{n}\right\| \\
& \leq \kappa_{1}+\tau\left\|y_{n}\right\|+\sigma \leq \kappa_{1}+\tau\left(\left\|x_{n}\right\|+\varepsilon_{n}\left\|v_{n}^{*}\right\|\right)+\sigma,
\end{aligned}
$$

for all $n$, where $\kappa_{1}$ is an upper bound for $\left\{\left\|w_{n}^{*}\right\|+\left\|u_{n}^{*}\right\|\right\}$. This gives the boundedness of $\left\{v_{n}^{*}\right\}$ and $\left\{y_{n}\right\}$. Since $x_{n} \rightarrow x_{0}$ as $n \rightarrow \infty, y_{n}=x_{n}-\varepsilon_{n} J^{-1}\left(v_{n}^{*}\right)$ and $\left\{v_{n}^{*}\right\}$ is bounded, it follows that $y_{n} \rightarrow x_{0}$ as $n \rightarrow \infty$. Assume without loss of generality that $C y_{n} \rightarrow g_{0}^{*}$ as $n \rightarrow \infty$. Since $y_{n}-x_{n} \rightarrow 0$ as $n \rightarrow \infty$, the quasimonotonicity of $S_{1}$ and $S_{2}$ implies

$$
\begin{aligned}
& \limsup _{n \rightarrow \infty}\left\langle v_{n}^{*}, y_{n}-x_{0}\right\rangle \\
&=-\liminf _{n \rightarrow \infty}\left\langle t_{n} w_{n}^{*}+\left(1-t_{n}\right) u_{n}^{*}+t_{n} C y_{n}, y_{n}-x_{0}\right\rangle \\
& \leq-\liminf _{n \rightarrow \infty}\left\langle t_{n} w_{n}^{*}+\left(1-t_{n}\right) u_{n}^{*}, y_{n}-x_{n}+x_{n}-x_{0}\right\rangle \\
& \quad-\lim _{n \rightarrow \infty}\left\langle t_{n} C y_{n}, y_{n}-x_{0}\right\rangle \\
& \leq-\liminf _{n \rightarrow \infty}\left\langle t_{n} w_{n}^{*}+\left(1-t_{n}\right) u_{n}^{*}, y_{n}-x_{n}\right\rangle \\
& \quad-\liminf _{n \rightarrow \infty}\left\langle t_{n} w_{n}^{*}+\left(1-t_{n}\right) u_{n}^{*}, x_{n}-x_{0}\right\rangle \\
&=-\liminf _{n \rightarrow \infty}\left\langle t_{n} w_{n}^{*}+\left(1-t_{n}\right) u_{n}^{*}, x_{n}-x_{0}\right\rangle \\
& \leq--\liminf _{n \rightarrow \infty} t_{n}\left\langle w_{n}^{*}, x_{n}-x_{0}\right\rangle \\
& \quad-\liminf _{n \rightarrow \infty}\left(1-t_{n}\right)\left\langle u_{n}^{*}, x_{n}-x_{0}\right\rangle \leq 0 .
\end{aligned}
$$

Therefore, we get

$$
\limsup _{n \rightarrow \infty}\left\langle v_{n}^{*}, y_{n}\right\rangle \leq\left\langle v_{0}^{*}, x_{0}\right\rangle .
$$

Since $\overline{D(T)}=X$, the result of Kobayashi and Otani [8] says that the family $\{t T\}_{t \in[0,1]}$ is a pseudomonotone homotopy of maximal monotone operators. By (i) of Definition 6, we conclude that $x_{0} \in D\left(T^{t_{0}}=t_{0} T\right), v_{0}^{*} \in t_{0} T x_{0}$, and $\left\langle v_{n}^{*}, y_{n}\right\rangle \rightarrow$ $\left\langle v_{0}^{*}, x_{0}\right\rangle$ as $n \rightarrow \infty$. Applying analogous arguments to those of the proof of (iv) along with the $\left(S_{+}\right)$condition on $S_{1}$ and $S_{2}$, one can easily verify that $x_{n} \rightarrow x_{0} \in D(T) \cap \partial G, C y_{n} \rightarrow C x_{0}$, $w_{n}^{*} \rightarrow w_{0}^{*} \in S_{1} x_{0}$, and $u_{n}^{*} \rightarrow u_{0}^{*} \in S_{2} x_{0}$ so that $v_{0}^{*}+t_{0} w_{0}^{*}+(1-$ $\left.t_{0}\right) u_{0}^{*}+t_{0} C x_{0}=0$; that is, $0 \in\left(t_{0}\left(T+S_{1}+C\right)+\left(1-t_{0}\right) S_{2}\right)(D(T) \cap$ $\partial G)$, which is impossible by the hypotheses. In conclusion, we have proved that $d\left(T_{\varepsilon}^{t}+t S_{1}+(1-t) S_{2}+t C J_{\varepsilon}, G, 0\right)$ is welldefined for all $t \in[0,1]$ and sufficiently small $\varepsilon>0$.

Finally, we shall show that $d\left(T_{\varepsilon}^{t}+t S_{1}+(1-t) S_{2}+t C J_{\varepsilon}, G, 0\right)$ is independent of $t \in[0,1]$ and $\varepsilon \in\left(0, \varepsilon_{0}\right]$. To this end, let $0<\varepsilon_{1}<\varepsilon_{2} \leq \varepsilon_{0}, q(t)=t \varepsilon_{1}+(1-t) \varepsilon_{2}, 0<t_{1}<t_{2} \leq 1$, and $\gamma_{t}=t t_{1}+(1-t) t_{2}, t \in[0,1]$. To complete the proof, we consider the homotopy operator

$$
\begin{array}{r}
N_{1}(t, x)=T_{q(t)}^{\gamma_{t}} x+t S_{1} x+(1-t) S_{2} x+t C J_{q(t)}^{t} x, \\
(t, x) \in[0,1] \times \bar{G} .
\end{array}
$$

It is sufficient to show that $\left\{N_{1}(t, \cdot)\right\}_{t \in[0,1]}$ is a homotopy of class $\left(S_{+}\right)$. For each $t \in[0,1]$, it is easy to see that $N_{1}(t, \cdot)$ : 
$X \rightarrow 2^{X^{*}}$ is bounded and of type $\left(S_{+}\right)$. Let $\left\{x_{n}\right\} \subset X$ be such that $x_{n} \rightarrow x_{0}, t_{n} \rightarrow t_{0}$ as $n \rightarrow \infty, w_{n}^{*} \in S_{1} x_{n}$, and $u_{n}^{*} \in S_{2} x_{n}$ so that $\lim \sup _{n \rightarrow \infty}\left\langle T_{q\left(t_{n}\right)}^{\gamma_{t_{n}}} x_{n}+g_{n}^{*}, x_{n}-x_{0}\right\rangle \leq 0$, where $g_{n}^{*}=$ $t_{n} w_{n}^{*}+\left(1-t_{n}\right) u_{n}^{*}+t_{n} C J_{q\left(t_{n}\right)}^{t_{n}} x_{n}$. Let

$$
\begin{aligned}
& z_{n}^{*}=T_{q\left(t_{n}\right)}^{\gamma_{t_{n}}} x_{n}, \\
& z_{n}=J_{q\left(t_{n}\right)}^{\gamma_{t_{n}}} x_{n}
\end{aligned}
$$

$\forall n$.

We show that $\left\{z_{n}^{*}\right\}$ and $\left\{z_{n}\right\}$ are bounded. Since $0<\varepsilon_{1} \leq$ $q\left(t_{n}\right) \leq \varepsilon_{2}$ and $0<t_{1} \leq \gamma_{t} \leq t_{2}$ for all $n$, we conclude from Lemma 4 that $\left\{z_{n}^{*}\right\}$ is bounded. Since $z_{n}=x_{n}-q\left(t_{n}\right) J^{-1}\left(z_{n}^{*}\right)$ for all $n$ and $\left\{z_{n}^{*}\right\},\left\{q\left(t_{n}\right)\right\}$ and $\left\{x_{n}\right\}$ are bounded, we get the boundedness of $\left\{z_{n}\right\}$. Since $C$ is compact, we assume without loss of generality that $C z_{n} \rightarrow h_{0}^{*}$ as $n \rightarrow \infty$. By the boundedness of $\left\{x_{n}\right\}, S_{1}$, and $S_{2}$, we assume, by passing into subsequences if necessary, that $x_{n} \rightarrow x_{0}, w_{n}^{*} \rightarrow w_{0}^{*}$, and $u_{n}^{*} \rightarrow u_{0}^{*}$ as $n \rightarrow \infty$. On the other hand, the pseudomonotonicity of $S_{1}$ and $S_{2}$ gives

$$
\liminf _{n \rightarrow \infty}\left\langle g_{n}^{*}, x_{n}-x_{0}\right\rangle \geq 0
$$

Consequently, we get

$$
\begin{aligned}
\limsup _{n \rightarrow \infty}\left\langle z_{n}^{*}, x_{n}-x_{0}\right\rangle \leq & \limsup _{n \rightarrow \infty}\left\langle z_{n}^{*}+g_{n}^{*}, x_{n}-x_{0}\right\rangle \\
& -\liminf _{n \rightarrow \infty}\left\langle g_{n}^{*}, x_{n}-x_{0}\right\rangle \\
\leq & -\liminf _{n \rightarrow \infty}\left\langle g_{n}^{*}, x_{n}-x_{0}\right\rangle \leq 0 .
\end{aligned}
$$

Since $T$ is positively homogeneous of order $\alpha>0$, it is not difficult to see that $T^{-1}: R(T) \rightarrow D(T)$ is maximal monotone and positively homogeneous of order $\alpha^{-1}>0$. It also holds that $(\lambda T)^{-1}(x)=T^{-1}((1 / \lambda)(x))=\lambda^{-1 / \alpha} T^{-1}(x)$ for all $x \in$ $R(T)$. In addition, we see that

$$
\begin{aligned}
z_{n}^{*} & =T_{q\left(t_{n}\right)}^{\gamma_{t_{n}}} x_{n}=\left(\gamma_{t_{n}} T\right)_{q\left(t_{n}\right)} x_{n} \\
& =\left(\left(\gamma_{t_{n}} T\right)^{-1}+q\left(t_{n}\right) J^{-1}\right)^{-1} x_{n} \\
& =\left(\frac{1}{\gamma_{t_{n}}^{1 / \alpha}} T^{-1}+q\left(t_{n}\right) J^{-1}\right)^{-1} x_{n} \\
& =\left(\frac{1}{\gamma_{t_{n}}^{1 / \alpha}}\left(T^{-1}+q\left(t_{n}\right) \gamma_{t_{n}}^{1 / \alpha} J^{-1}\right)\right)^{-1} x_{n} \\
& =\gamma_{t_{n}}^{1 / \alpha}\left(T^{-1}+q\left(t_{n}\right) \gamma_{t_{n}}^{1 / \alpha} J^{-1}\right)^{-1} x_{n} \\
& =\gamma_{t_{n}}^{1 / \alpha} T_{q\left(t_{n}\right) \gamma_{t_{n}}^{1 / \alpha}}\left(x_{n}\right) \quad \forall n .
\end{aligned}
$$

In fact, it is true that, $T_{\varepsilon}^{a}(x)=a^{1 / \alpha} T_{\varepsilon a^{1 / \alpha}}(x)$ for all $x \in X, \varepsilon>0$ and $a>0$. For each $n$, letting $\lambda_{n}=\gamma_{t_{n}}^{1 / \alpha}$, we get

$$
\begin{aligned}
\left\langle z_{n}^{*}, x_{n}-x_{0}\right\rangle= & \left\langle\lambda_{n} T_{q\left(t_{n}\right) \lambda_{n}} x_{n}, x_{n}-x_{0}\right\rangle \\
= & \lambda_{n}\left\langle T_{q\left(t_{n}\right) \lambda_{n}} x_{n}, x_{n}-x_{0}\right\rangle \\
= & \lambda_{n}\left\langle T_{q\left(t_{n}\right) \lambda_{n}} x_{n}-T_{q\left(t_{n}\right) \lambda_{n}} x_{0}, x_{n}-x_{0}\right\rangle \\
& +\lambda_{n}\left\langle T_{q\left(t_{n}\right) \lambda_{n}} x_{0}, x_{n}-x_{0}\right\rangle \quad \forall n .
\end{aligned}
$$

Since $(0, \infty) \times X \ni(t, x) \rightarrow T_{t} x$ is continuous, it follows that $T_{q\left(t_{n}\right) \lambda_{n}} x_{0} \rightarrow T_{q\left(t_{0}\right) \lambda_{0}} x_{0}$ as $n \rightarrow \infty$, where $\lambda_{n} \rightarrow \lambda_{0}>0$ as $n \rightarrow \infty$. By the monotonicity of $T_{q\left(t_{n}\right) \lambda_{n}}$ for all $n$, we have

$$
\liminf _{n \rightarrow \infty}\left\langle z_{n}^{*}, x_{n}-x_{0}\right\rangle \geq 0
$$

that is,

$$
\begin{aligned}
0 & \leq \liminf _{n \rightarrow \infty}\left\langle z_{n}^{*}, x_{n}-x_{0}\right\rangle \leq \limsup _{n \rightarrow \infty}\left\langle z_{n}^{*}, x_{n}-x_{0}\right\rangle \\
& \leq 0
\end{aligned}
$$

which implies $\left\langle z_{n}^{*}, x_{n}\right\rangle \rightarrow\left\langle z_{0}^{*}, x_{0}\right\rangle$ as $n \rightarrow \infty$. Consequently, we arrive at

$$
\begin{array}{r}
\limsup _{n \rightarrow \infty}\left\langle w_{n}^{*}, x_{n}-x_{0}\right\rangle \leq 0 \\
\text { or } \limsup _{n \rightarrow \infty}\left\langle u_{n}^{*}, x_{n}-x_{0}\right\rangle \leq 0 .
\end{array}
$$

Since both $S_{1}$ and $S_{2}$ are bounded and of type $\left(S_{+}\right)$, it follows that $x_{n} \rightarrow x_{0}$ as $n \rightarrow \infty$. As a result of this, we get

$$
\begin{aligned}
& z_{n}^{*}=\lambda_{n} T_{q\left(t_{n}\right) \lambda_{n}} x_{n} \longrightarrow \lambda_{0} T_{q\left(t_{0}\right) \lambda_{0}} x_{0}=z_{0}^{*}, \\
& z_{n}=x_{n}-q\left(t_{n}\right) J^{-1}\left(z_{n}^{*}\right) \longrightarrow \\
& x_{0}-q\left(t_{0}\right) \lambda_{0} J^{-1}\left(T_{q\left(t_{0}\right) \lambda_{0}} x_{0}\right)=z_{0}=J_{q\left(t_{0}\right)}^{\lambda_{0}} x_{0}
\end{aligned}
$$

as $n \rightarrow \infty$; that is, we have $\lim _{\sup _{n \rightarrow \infty}}\left\langle z_{n}^{*}, z_{n}\right\rangle \leq\left\langle z_{0}^{*}, z_{0}\right\rangle$. Since $\{t T\}_{t \in[0,1]}$ is a pseudomonotone homotopy of maximal monotone operators, it follows that $z_{0} \in D\left(t_{0} T\right)$ and $z_{0}^{*} \epsilon$ $t_{0} T\left(z_{0}\right)$. In conclusion, we obtain that $x_{0} \in \partial G, w_{0}^{*} \in S_{1} x_{0}$, $u_{0}^{*} \in S_{2} x_{0}, z_{0} \in D(T)$, and

$$
\begin{aligned}
& z_{0}^{*}+t_{0} w_{0}^{*}+\left(1-t_{0}\right) u_{0}^{*}+t_{0} C z_{0} \\
& \quad \in T_{q\left(t_{0}\right)}^{\gamma_{t_{0}}} x_{0}+t_{0} S_{1} x_{0}+\left(1-t_{0}\right) S_{2} x_{0}+C z_{0} .
\end{aligned}
$$

Therefore, for any $c_{0} \in(0,1]$, the family $\left\{N_{1}(t, \cdot)\right\}_{t \in\left[c_{0}, 1\right]}$ is a homotopy of class $\left(S_{+}\right)$. Thus, $d\left(N_{1}(t, \cdot), G, 0\right)$ is independent of $t \in(0,1]$ and $\varepsilon \in\left(0, \varepsilon_{0}\right]$; that is,

$$
\begin{aligned}
d\left(N_{1}(t, \cdot), G, 0\right) & =d\left(N_{1}(1, \cdot), G, 0\right) \\
& =d\left(T_{\varepsilon_{1}}+S_{1}+C J_{\varepsilon_{1}}, G, 0\right)
\end{aligned}
$$


On the other hand, by the definition of $d$, we have that

$$
\begin{aligned}
d & (N(t, \cdot), G, 0) \\
\quad & =d\left(T_{\varepsilon}^{t}+t S_{1}+(1-t) S_{2}+C J_{\varepsilon}^{t}, G, 0\right)
\end{aligned}
$$

is independent of $t \in(0,1]$ and $\varepsilon \in\left(0, \varepsilon_{0}\right]$. In particular, for $t=1$, we have $d(N(t, \cdot), G, 0)=d\left(T_{\varepsilon}+S_{1}+C J_{\varepsilon}, G, 0\right)$ for all $t \in(0,1]$. But, for $t=0$, we see that $N(0, x)=S_{2} x$ for all $x \in X$. To complete the proof, it is sufficient to show that

$$
d\left(T_{\varepsilon}+S_{1}+C J_{\varepsilon}, G, 0\right)=d\left(S_{2}, G, 0\right) .
$$

For each $\varepsilon>0$, we consider the homotopy

$$
\begin{aligned}
N_{\varepsilon}(t, x)=T_{\varepsilon} x+t\left(S_{1} x+C J_{\varepsilon} x\right)+(1-t) & S_{2} x, \\
& (t, x) \in[0,1] \times \bar{G} .
\end{aligned}
$$

Suppose that there exist $\varepsilon_{n} \downarrow 0^{+}, t_{n} \in[0,1], x_{n} \in \partial G, w_{n}^{*} \in$ $S_{1} x_{n}$, and $u_{n}^{*} \in S_{2} x_{n}$ such that

$$
T_{\varepsilon_{n}} x_{n}+t_{n}\left(w_{n}^{*}+C J_{\varepsilon_{n}} x_{n}\right)+\left(1-t_{n}\right) u_{n}^{*}=0 \quad \forall n .
$$

We assume without loss of generality that $t_{n} \rightarrow t_{0} \in[0,1]$, $x_{n} \rightarrow x_{0}, w_{n}^{*} \rightarrow w_{0}^{*}$, and $u_{n}^{*} \rightarrow u_{0}^{*}$ as $n \rightarrow \infty$. By the $\Gamma_{\sigma}^{\tau}$ condition on $C$, we get

$$
\begin{aligned}
\left\|T_{\varepsilon_{n}} x_{n}\right\| & \leq t_{n} \tau\left\|J_{\varepsilon_{n}} x_{n}\right\|+\kappa_{2} \\
& \leq \tau\left\|x_{n}-\varepsilon_{n} J^{-1}\left(T_{\varepsilon_{n}} x_{n}\right)\right\|+\kappa_{2} \\
& \leq \tau\left\|x_{n}\right\|+\tau \varepsilon_{n}\left\|T_{\varepsilon_{n}} x_{n}\right\|+\kappa_{2} \quad \forall n,
\end{aligned}
$$

where $\kappa_{2}$ is an upper bound for the sequence $\left\{\sigma+\left\|w_{n}^{*}\right\|+\right.$ $\left.\left\|u_{n}^{*}\right\|\right\}$. This shows the boundedness of $\left\{T_{\varepsilon_{n}} x_{n}\right\}$ and $\left\{J_{\varepsilon_{n}} x_{n}\right\}$. By the maximality of $T$ along with Lemma 5 , the compactness of $C$, and the $\left(S_{+}\right)$condition on $S_{1}$ and $S_{2}$ and analogous arguments to those in the proof of Theorem 9, we conclude that $x_{0} \in D(T) \cap \partial G, v_{0}^{*} \in T x_{0}, w_{0}^{*} \in S_{1} x_{0}$, and $u_{0}^{*} \in S_{2} x_{0}$ so that $v_{0}^{*}+t_{0}\left(w_{0}^{*}+C x_{0}\right)+\left(1-t_{0}\right) u_{0}^{*}=0$; that is, $0 \in$ $\left(T+t_{0}\left(S_{1}+\mathrm{C}\right)+\left(1-t_{0}\right) S_{2}\right)(D(T) \cap \partial G)$. However, this is impossible. In addition, the boundary condition on $M$ in (v) implies that $\left\{N_{\varepsilon}(t, \cdot)\right\}_{t \in[0,1]}$ is an admissible homotopy; that is, $d\left(T_{\varepsilon}+S_{1}+C J_{\varepsilon}, G, 0\right)=d\left(T_{\varepsilon}+S_{2}, G, 0\right)$ for all $\varepsilon \in\left(0, \varepsilon_{0}\right]$. Since $0 \in G$ and $S_{2}$ satisfies the condition $\left\langle u^{*}, x\right\rangle \geq\|x\|^{2}$ for all $x \in X$ and $u^{*} \in S_{2} x$ and $0 \in T(0)$, it follows that $0 \neq t\left(T_{\varepsilon}+S_{2} x\right)+(1-t) S_{2} x$ for all $x \in \partial G$ and $t \in[0,1]$ and $d\left(t\left(T_{\varepsilon}+S_{2}\right)+(1-t) S_{2}, G, 0\right)$ is independent of $t \in[0,1]$. In particular, $d\left(T_{\varepsilon}+S_{2}, G, 0\right)=d\left(S_{2}, G, 0\right)$ for all $\varepsilon \in\left(0, \varepsilon_{0}\right]$. Therefore, we conclude that $d(N(t, \cdot), G, 0)$ is independent of all $t \in[0,1]$. This completes the proof.

2.3. Degree Theory for $T+C+S$ with $S$ Pseudomonotone. In this section we present a generalization of the theory developed in the previous section for operators of type $T+C+S$, where $S: X \rightarrow 2^{X^{*}}$ is bounded pseudomonotone and T, $C$ satisfy the conditions of Section 2.1. For each $\varepsilon>0$, it is well-known that $S+\varepsilon J$ is bounded and of type $\left(S_{+}\right)$. As a result of this, we may apply the arguments used in the proof of Lemma 7 to show that $d\left(T+S+C+\varepsilon J, G, f^{*}\right)$ is well-defined and constant for all sufficiently small $\varepsilon>0$ provided that $f^{*} \notin$ $\overline{(T+S+C)(D(T) \cap \partial G)}$, where $d$ is given in Definition 6 . We thus give the following definition.

Definition 10. Let $G$ be a nonempty, bounded, and open subset of $X, T: X \supset D(T) \rightarrow 2^{X^{*}}$ be maximal monotone, $S: X \rightarrow 2^{X^{*}}$ be bounded pseudomonotone, and $C: D(C) \rightarrow$ $X^{*}$ be compact with $D(T) \subseteq D(C)$ and belonging to the class $\Gamma_{\sigma}^{\tau}$. Assume, further, that $f^{*} \notin(T+S+C)(D(T) \cap \partial G)$. Then the degree mapping $d$ for $T+S+C$ at $f^{*} \in X^{*}$ with respect to $G$ is defined by

$$
d\left(T+S+C, G, f^{*}\right)=\lim _{\varepsilon \downarrow 0^{+}} d\left(T+C+S+\varepsilon J, G, f^{*}\right),
$$

where $d\left(T+C+S+\varepsilon J, G, f^{*}\right)$ denotes the degree mapping constructed in Section 2.1.

The following theorem gives some basic properties and homotopy invariance results analogous to those of Theorem 9.

Theorem 11. Let $G$ be a nonempty, bounded, and open subset of $X$. Let $T: X \supset D(T) \rightarrow 2^{X^{*}}$ be maximal monotone, $S:$ $X \rightarrow 2^{X^{*}}$ be bounded pseudomonotone, and $C: X \supseteq D(C) \rightarrow$ $X^{*}$ be compact with $D(T) \subseteq D(C)$ and belonging to the class $\Gamma_{\sigma}^{\tau}$. Then the following properties hold:

(i) (Normalization) $d(J, G, 0)=1$ if $0 \in G$ and $d(J, G, 0)=0$ if $0 \notin \bar{G}$.

(ii) (Existence) if $f^{*} \notin \overline{(T+S+C)(D(T) \cap \partial G)}$ and $d(T+$ $\left.S+C, G, f^{*}\right) \neq 0$, then $f^{*} \in \overline{(T+S+C)(D(T) \cap G)}$. If $T+S$ is of type $(S)$, then $f^{*} \in(T+C+S)(D(T) \cap G)$.

(iii) (Decomposition) let $G_{1}$ and $G_{2}$ be nonempty and disjoint open subsets of $G$ such that $f^{*} \notin$ $\overline{(T+S+C)\left(D(T) \cap\left(\bar{G} \backslash\left(G_{1} \cup G_{2}\right)\right)\right)}$. Then

$$
\begin{aligned}
d\left(T+S+C, G, f^{*}\right)= & d\left(T+S+C, G_{1}, f^{*}\right) \\
& +d\left(T+S+C, G_{2}, f^{*}\right) .
\end{aligned}
$$

(iv) (Translation invariance) let $f^{*} \notin \overline{(T+S+C)(D(T) \cap \partial G)}$. Then we have

$$
d\left(T+S+C-f^{*}, G, 0\right)=d\left(T+S+C, G, f^{*}\right) .
$$

(v) Let $M(t, x)=T x+t\left(S_{1} x+C x\right)+(1-t) S_{2} x$, $(t, x) \in[0,1] \times(D(T) \cap \bar{G})$, and $S_{i}: X \rightarrow 2^{X^{*}}(i=$ $1,2)$ be bounded pseudomonotone such that $0 \notin$ $\overline{M(t, D(T) \cap \partial G)}$ for all $t \in[0,1]$. Then $d(M(t, \cdot), G, 0)$ is independent of $t \in[0,1]$.

(vi) Let $N(t, x)=t\left(T x+S_{1} x+C x\right)+(1-t) S_{2} x,(t, x) \epsilon$ $[0,1] \times(D(T) \cap \bar{G})$, and $T: X \supset D(T) \rightarrow X^{*}$ be densely defined maximal monotone and positively homogeneous of order $\alpha>0, S_{1}: X \rightarrow 2^{X^{*}}$ be 
bounded pseudomonotone, $S_{2}: X \rightarrow 2^{X^{*}}$ be bounded and of type $\left(S_{+}\right)$, and $0 \notin \overline{N(t, D(T) \cap \partial G)}$ for all $t \in[0,1]$. Assume, further, that $0 \notin \overline{M(t, D(T) \cap \partial G)}$ for all $t \in[0,1]$. Then $d(N(t, \cdot), G, 0)$ is independent of $t \in[0,1]$.

Proof. The proofs for (i) through (iv) follow as in the analogous items in the proof of Theorem 9. We shall give sketches of the proofs of (v) and (vi). To prove (v), for each $\varepsilon>0$, we consider the homotopy inclusion

$$
\begin{aligned}
M_{\varepsilon}(t, x)=T x+t\left(S_{1} x+C x\right) & +(1-t) S_{2} x+\varepsilon J x, \\
(t, x) & \in[0,1] \times(D(T) \cap \bar{G}) .
\end{aligned}
$$

Following the arguments used in the proof of (v) of Theorem 9 , it can be shown that there exists $\varepsilon_{0}>0$ such that $0 \notin M_{\varepsilon}(t, D(T) \cap \partial G)$ for all $t \in[0,1]$ and $\varepsilon \in\left(0, \varepsilon_{0}\right]$. Otherwise, we would get $0 \in \overline{M\left(t_{0}, D(T) \cap \partial G\right)}$ for some $t_{0} \in[0,1]$, which is impossible. On the other hand, for each $\varepsilon \in\left(0, \varepsilon_{0}\right]$, we see that

$$
\begin{aligned}
M_{\varepsilon}(t, x)= & T x+t\left(S_{1} x+C x+\varepsilon J x\right) \\
& +(1-t)\left(S_{2} x+\varepsilon J x\right), \\
& (t, x) \in[0,1] \times(D(T) \cap \bar{G}) .
\end{aligned}
$$

Since $S_{1}+\varepsilon J$ and $S_{2}+\varepsilon J$ are bounded operators of type $\left(S_{+}\right)$, the proof of $(\mathrm{v})$ of Theorem 9 implies that $d\left(M_{\mathcal{\varepsilon}}(t, \cdot), G, 0\right)$ is independent of $t \in[0,1]$; that is,

$$
\begin{aligned}
d\left(M_{\varepsilon}(t, \cdot), G, 0\right) & =d\left(T+S_{1}+C+\varepsilon J, G, 0\right) \\
& =d\left(T+S_{1}+C, G, 0\right)
\end{aligned}
$$

for all $t \in[0,1]$ and $\varepsilon \in\left(0, \varepsilon_{0}\right]$. As a result of this, we get

$$
\begin{aligned}
d(M(t, \cdot), G, 0) & =\lim _{\varepsilon \downarrow 0^{+}} d\left(M_{\varepsilon}(t, \cdot), G, 0\right) \\
& =d\left(T+S_{1}+C, G, 0\right)
\end{aligned}
$$

for all $t \in[0,1]$. This proves that $d(M(t, \cdot), G, 0)$ is independent of $t \in[0,1]$ provided that $0 \notin \overline{M(t, D(T) \cap \partial G)}$ for all $t \in[0,1]$. The proof of (vi) can be completed in analogous manner. The details are omitted here.

\section{An Existence Theorem}

As a consequence of the degree theory developed in Section 2, the following theorem gives a new existence result on the solvability of operator inclusions of the type $T u+S u+C u \ni f^{*}$ in $D(T)$ provided that $T+S$ is of type $(S)$ or $S$ is bounded of type $\left(S_{+}\right)$.

Theorem 12. Let $T: X \supset D(T) \rightarrow 2^{X^{*}}$ be maximal monotone with $0 \in T(0), S: X \rightarrow 2^{X^{*}}$ be bounded pseudomonotone, and $C: X \supseteq D(C) \rightarrow X^{*}$ be compact with $D(T) \subseteq D(C)$ and belonging to the class $\Gamma_{\sigma}^{\tau}$. Let $f^{*} \in X^{*}$. Assume, further, that there exists $R>0$ such that

$$
\left\langle v^{*}+w^{*}+C x-f^{*}, x\right\rangle>0
$$

for all $x \in D(T) \cap \partial B_{R}(0), v^{*} \in T x$, and $w^{*} \in S x$. Then $f^{*} \in$ $\overline{(T+S+C)\left(D(T) \cap B_{R}(0)\right)}$. Furthermore, $\overline{R(T+S+C)}=X^{*}$ provided that $T+S+C$ is coercive.

Proof. Let $\varepsilon>0$. We shall show that $0 \notin K_{\varepsilon}\left(t, D(T) \cap \partial B_{R}(0)\right)$ for all $t \in[0,1]$, where

$$
\begin{aligned}
K_{\varepsilon}(t, x)= & T x+t\left(S x+C x+\varepsilon J x-f^{*}\right) \\
+(1-t)(\varepsilon J x), & \\
& (t, x) \in[0,1] \times\left(D(T) \cap \bar{B}_{R}(0)\right) .
\end{aligned}
$$

Since $0 \in T(0)$, by using the boundary condition on $T+S+C$, we see that

$$
\begin{aligned}
& \left\langle t\left(v^{*}+w^{*}+C x-f^{*}\right), x\right\rangle+\left\langle(1-t) v^{*}+\varepsilon J x, x\right\rangle \\
& \quad \geq\left\langle(1-t) v^{*}+\varepsilon J x, x\right\rangle \\
& \quad=(1-t)\left\langle v^{*}, x\right\rangle+\varepsilon\langle J x, x\rangle \geq \varepsilon\|x\|^{2}=\varepsilon R^{2}>0
\end{aligned}
$$

for all $t \in[0,1], x \in D(T) \cap \partial B_{R}(0), v^{*} \in T x$, and $w^{*} \in S x$; that is, for each $\varepsilon>0$, it follows that $0 \notin K_{\varepsilon}\left(t, D(T) \cap \partial B_{R}(0)\right)$ for all $t \in[0,1]$. Since $\varepsilon J$ and $S+\varepsilon J$ are continuous, bounded, and of type $\left(S_{+}\right),(\mathrm{v})$ of Theorem 9 implies that $\left\{K_{\varepsilon}(t, \cdot)\right\}_{t \in[0,1]}$ is an admissible homotopy. Therefore, for each $\varepsilon>0$, we obtain

$$
\begin{aligned}
d\left(K_{\varepsilon}(t, \cdot), B_{R}(0), 0\right)=d\left(\varepsilon J, B_{R}(0), 0\right)= & \\
& \forall t \in[0,1] ;
\end{aligned}
$$

that is, $d\left(T+S+C+\varepsilon J, B_{R}(0), f^{*}\right)=1$. By (ii) of Theorem 9, we conclude that $f^{*} \in(T+S+C+\varepsilon J)\left(D(T) \cap B_{R}(0)\right)$; that is, for each $\varepsilon_{n} \downarrow 0^{+}$, there exist $x_{n} \in D(T) \cap B_{R}(0), v_{n}^{*} \in T x_{n}$, and $w_{n}^{*} \in S x_{n}$ such that

$$
v_{n}^{*}+w_{n}^{*}+C x_{n}+\varepsilon_{n} J x_{n}=f^{*} \quad \forall n .
$$

Since $\left\{x_{n}\right\}$ is bounded, we have $\varepsilon_{n} J x_{n} \rightarrow 0$ as $n \rightarrow \infty$, which implies that $f^{*} \in \overline{(T+S+C)\left(D(T) \cap B_{R}(0)\right)}$. If $T+S+C$ is coercive, then for each $f^{*} \in X^{*}$ there exists $R=R\left(f^{*}\right)>$ 0 such that the boundary condition holds. This implies that $f^{*} \in \overline{(T+S+C)\left(D(T) \cap B_{R}(0)\right)}$. Since $f^{*} \in X^{*}$ is arbitrary, we conclude that $\overline{R(T+S+C)}=X^{*}$. The proof is complete.

The arguments used in the proof of Theorem 12 gives the following existence result on the surjectivity of $T+S+C$ provided that either $S$ is bounded and of type $\left(S_{+}\right)$or $T+S$ is of type $(S)$.

Corollary 13. Let $T: X \supset D(T) \rightarrow 2^{X^{*}}$ be maximal monotone with $0 \in T(0), S: X \rightarrow 2^{X^{*}}$, and $C: D(C) \rightarrow X^{*}$ be compact with $D(T) \subseteq D(C)$ and belonging to the class $\Gamma_{\sigma}^{\tau}$. Let $f^{*} \in X^{*}$. Assume, further, that $T+S+C$ is coercive. Then $T+S+C$ is surjective provided that $S$ is bounded of type $\left(S_{+}\right)$ or $T+S$ is of type $(S)$. 
Proof. Let $f^{*} \in X^{*}$. Suppose $T+S+C$ is coercive; that is, there exists $\phi:[0, \infty) \rightarrow(-\infty, \infty)$ and $\phi(t) \rightarrow \infty$ as $t \rightarrow \infty$ such that

$$
\begin{aligned}
\left\langle v^{*}+w^{*}+C x, x\right\rangle \geq & \phi(\|x\|)\|x\| \\
& \forall x \in D(T), v^{*} \in T x, w^{*} \in S x .
\end{aligned}
$$

Then, there exists $R=R\left(f^{*}\right)>0$ such that

$$
\left\langle v^{*}+w^{*}+C x-f^{*}, x\right\rangle>0
$$

for all $x \in D(T) \cap \partial B_{R}(0), v^{*} \in T x$, and $w^{*} \in S x$. Assume that $T+S$ is of type $(S)$. By Theorem 12 , we conclude that $f^{*} \in$ $\overline{(T+S+C)\left(D(T) \cap B_{R}(0)\right)}$; that is, there exists $x_{n} \in D(T) \cap$ $B_{R}(0), v_{n}^{*} \in T x_{n}$, and $w_{n}^{*} \in S x_{n}$ such that $v_{n}^{*}+w_{n}^{*}+C x_{n} \rightarrow f^{*}$ as $n \rightarrow \infty$. Since $C$ is compact, we assume without loss of generality that $C x_{n} \rightarrow g_{0}^{*}$ as $n \rightarrow \infty$; that is, $v_{n}^{*}+w_{n} \rightarrow f^{*}-$ $g_{0}^{*}$ as $n \rightarrow \infty$. Since $T+S$ is of type $(S)$, it follows that $x_{n} \rightarrow x_{0}$ as $n \rightarrow \infty$. By the maximality of $T$ along with Lemma 5, the continuity of $C$ and generalized pseudomonotonicity of $S$, and the arguments used in the proof of Lemma 7, we conclude that $x_{0} \in D(T) \cap B_{R}(0)$ and $f^{*} \in T x_{0}+S x_{0}+C x_{0}$; that is, $f^{*} \in(T+S+C)\left(D(T) \cap B_{R}(0)\right)$. Since $f^{*} \in X^{*}$ is arbitrary, we conclude that $T+S+C$ is surjective. The case when $S$ is bounded and of type $\left(S_{+}\right)$can be reached by following analogous arguments. The details are omitted here.

Theorem 12 is a new result and Corollary 13 gives a surjectivity result for operators of the type $T+S+C$. For further existence results involving operators of the type $T+$ $S$, the reader is referred to Kenmochi [17], Le [18], and Asfaw [19]. For various examples on pseudomonotone and quasimonotone operators, we cite the paper due to Mustonen [20].

\section{An Example}

Let $H=L^{2}(0, T ; V)$ and $V=W_{0}^{1,2}(\Omega)$. It is well-known that $H$ and $V$ are real Hilbert spaces with duality pairing between $u \in H$ and $v \in H$ denoted by $\langle u, v\rangle$ which is given by

$$
\langle u, v\rangle=\int_{0}^{T}\langle u(t), v(t)\rangle_{V} d t, \quad u \in H, v \in H,
$$

where $\langle u(t), v(t)\rangle_{V}$ denotes the duality pairing between $u(t) \epsilon$ $V$ and $v(t) \in V, t \in[0, T]$; that is, the norm of $u \in H$ is given by $\|u\|^{2}=\int_{0}^{T}\|u(t)\|_{V}^{2} d t$, where $\|u(t)\|_{V}$ denotes the norm of $u(t)$ in $V$. We shall apply the existence theorem(s) derived with the aid of the degree theory developed in this paper to establish existence of weak solution(s) in $H$ for nonlinear problem given by

$$
\begin{aligned}
& \frac{\partial u}{\partial t}-\sum_{i=1}^{N} \frac{\partial}{\partial x_{i}} a_{i}(x, t, u, \nabla u)+g(x, t, u, \nabla u)=f(x, t) \\
& u(x, t)=0, \quad(x, t) \in \partial \Omega \times(0, T) \in Q \\
& u(x, 0)=u(x, T), \quad u_{t} \in H, x \in \Omega,
\end{aligned}
$$

where $Q=\Omega \times(0, T)$ and $f \in L^{2}(Q)$ and the functions $a_{i}$ $(i=1,2, \ldots, N)$ and $g$ satisfy the following measurability and sublinearity conditions:

$\left(C_{1}\right) a_{i}(x, t, \eta, \zeta)(i=1,2, \ldots, N)$ is Carathèodory function; that is, $(x, t) \mapsto a_{i}(x, t, \eta, \zeta)$ is measurable for almost all $(\eta, \zeta) \in \mathbb{R}^{N}$ and $(\eta, \zeta) \mapsto a_{i}(x, t, \eta, \zeta)$ is continuous for almost all $(x, t) \in \Omega \times[0, T]$. Assume, further, that there exist $c_{1}>0$ and $k_{1} \in L^{2}(Q)$ such that

$$
\left|a_{i}(x, \eta, \zeta)\right| \leq c_{1}(|\eta|+|\zeta|)+k_{1}(x, t)
$$

for all $(x, t) \in \Omega \times[0, T]$ and $\xi \in \mathbb{R}^{N}$.

$\left(C_{2}\right)$ There exists $c_{2}>0$ such that $\sum_{i=1}^{N} a_{i}(x, \eta, \zeta) \zeta_{i} \geq c_{2}|\zeta|^{2}$ for all $(x, t) \in Q,(\eta, \zeta) \in \mathbb{R} \times \mathbb{R}^{N}$.

$\left(C_{3}\right) g: Q \times \mathbb{R} \rightarrow \mathbb{R}$ is Carathèodory function and there exist $c_{3} \geq 0$ and $k_{2} \in L^{2}(Q)$ such that

$$
\begin{aligned}
& |g(x, t, \eta, \zeta)| \leq c_{3}|\eta|+k_{2}(x, t), \\
& g(x, \eta, \zeta) \eta \geq|\eta|^{2}
\end{aligned}
$$

for all $(x, t) \in Q, \eta \in \mathbb{R}$ and $\zeta \in \mathbb{R}^{N}$.

A weak solution $u \in H$ is understood as follows.

Definition 14. A function $u=u(x, t)$ is a weak solution of $(82)$ if $u \in H$ and $u_{t} \in H$ such that the following are satisfied:

(i) $u(x, t)=0 \quad \forall x \in \Omega$;

(ii) $u(x, 0)=u(x, T) \quad \forall x \in \partial \Omega$;

$$
\text { (iii) } \begin{aligned}
\left\langle u^{\prime}, \phi\right\rangle+\sum_{i=1}^{N} \int_{Q} a_{i}(x, t, u, \nabla u) \frac{\partial \phi}{\partial x_{i}} d x d t \\
+\int_{Q}(g(x, t, u, \nabla u)-f(x, t)) \phi(x, t) d x d t=0
\end{aligned}
$$

$\forall \phi \in H$

where $u^{\prime}$ is understood in the sense of distributions; that is,

$$
\begin{aligned}
\int_{0}^{T} u^{\prime}(t) \psi(t) d t & =-\int_{0}^{T} u(t) \psi^{\prime}(t) d t \quad \forall \psi \\
& \in \mathrm{C}_{0}^{\infty}(0, \mathrm{~T}) .
\end{aligned}
$$

Next we give the following theorem.

Theorem 15. Let $f \in L^{2}(Q)$. Assume that conditions $\left(C_{1}\right)$ through $\left(C_{3}\right)$ are satisfied. Then (82) admits at least one-weak solution.

Proof. Let $S: H \rightarrow H$ be given by

$$
\langle S u, v\rangle=\sum_{i=1}^{N} \int_{Q} a_{i}(x, t, u, \nabla u) \frac{\partial v}{\partial x_{i}} d x d t,
$$


By using $\left(C_{1}\right)$ and $\left(C_{2}\right)$, it is well-known that $S$ is bounded continuous of type $\left(S_{+}\right)$. For the proof of these facts and other relevant properties of pseudomonotone and $\left(S_{+}\right)$type differential operators, the reader is referred to the papers by Browder [21], Berkovits and Mustonen [22], Hu and Papageorgiou [7], Landes and Mustonen [23], and the references therein. Let $C: H \supseteq D(C) \rightarrow H$ be defined by

$$
\langle C u, v\rangle=\int_{Q} g(x, t, u, \nabla u) v(x, t) d x d t, \quad v \in H,
$$

where $u \in D(C)=\left\{y \in H: y^{\prime} \in H\right\}$ and $L: H \supseteq D(L) \rightarrow H$ such that $\langle L u, v\rangle=\int_{0}^{T}\left\langle u^{\prime}(t), v(t)\right\rangle d t, v \in H$, where $u \in$ $D(L)=\left\{y \in H: y^{\prime} \in H, y(0)=y(T)\right\}$, that is, $D(L) \subseteq D(C)$. It is well-known that $L$ is a densely defined maximal monotone operator. The proof of this result is due to Brèzis which can be found in the book by Zeidler [13, Theorem 32. L, pp. 897-899]. Since $D(C)$ is compactly embedded in $L^{2}(Q)$, it is known that $C$ is a completely continuous operator; that is, $C$ is a compact operator. Further reference on operators of the type $C$ and existence results for parabolic problems, the reader is referred to the recent book due to Carl et al. [24]. Next we shall use Theorem 12 using the compact operator $C$, the maximal monotone operator $L$, and the $\left(S_{+}\right)$operator $S$. It remains to show that $C$ lies in $\Gamma_{\sigma}^{\tau}$ and for each $f^{*} \in H$, there exists $R=R\left(f^{*}\right)>0$ such that $\left\langle L u+S u+C u-f^{*}, u\right\rangle>0$ for all $u \in D(L) \cap \partial B_{R}(0)$. To this end, by applying condition $\left(C_{3}\right)$, Hòlder's inequality and observing that $\|y(t)\|_{L^{2}(\Omega)} \leq\|y(t)\|_{V}$ for all $y \in H$ and $t \in[0,1]$, we see that

$$
\begin{aligned}
& |\langle C u, v\rangle|=\left|\int_{0}^{T}\left(\int_{\Omega} g(x, t, u, \nabla u) v(x, t) d x\right) d t\right| \\
& \leq \int_{0}^{T}\left(\int_{\Omega}|g(x, t, u, \nabla u)||v(x, t)| d x\right) d t \\
& \leq c_{3} \int_{0}^{T}\left(\int_{\Omega}(|u(t)|+|\nabla u(t)|)|v(x, t)| d x\right) d t \\
& +\int_{Q}\left|k_{2}(x, t)\right||v(x, t)| d x d t \\
& \leq c_{3}\left(\int_{0}^{T}\|u(t)\|_{L^{2}(\Omega)}\|v(t)\|_{L^{2}(\Omega)} d t\right) \\
& +c_{3}\left(\int_{0}^{T}\|\nabla u(t)\|_{L^{2}(\Omega)}\|v(t)\|_{L^{2}(\Omega)} d t\right) \\
& +\int_{0}^{T}\left\|k_{2}(t)\right\|_{L^{2}(\Omega)}\|v(t)\|_{L^{2}(\Omega)} d t \\
& \leq c_{3}\left(\int_{0}^{T}\|u(t)\|_{V}\|v(t)\|_{V} d t\right) \\
& +c_{3}\left(\int_{0}^{T}\|\nabla u(t)\|_{V}\|v(t)\|_{V} d t\right) \\
& +\int_{0}^{T}\left\|k_{2}(t)\right\|_{L^{2}(\Omega)}\|v(t)\|_{V} d t \\
& \leq 2 c_{3}\|u\|\|v\|+\left\|k_{2}\right\|_{L^{2}(\mathrm{Q})}\|v\| \quad \forall u \in H, \quad v \in H ;
\end{aligned}
$$

that is, we get that

$$
\langle C u, v\rangle \leq 2 c_{3}\|u\|\|v\|+\left\|k_{2}\right\|_{L^{2}(Q)}\|v\|
$$

for all $u \in H$ and $v \in H$. Consequently, taking supremum overall $v \in H$ with $\|v\| \leq 1$, we conclude that $\|C u\| \leq \tau\|u\|+\sigma$ for all $u \in H$, where $\tau=2 c_{3}$ and $\sigma=\left\|k_{2}\right\|_{L^{2}(Q)}$; that is, $C$ belongs to $\Gamma_{\sigma}^{\tau}$. Next we show the boundary condition in Theorem 12. To this end, by using conditions $\left(C_{1}\right)$ through $\left(C_{3}\right)$ and monotonicity of $L(\langle L u, u\rangle \geq 0$ for all $u \in D(L))$, we get

$$
\begin{aligned}
\langle L u+S u+C u, u\rangle & \\
\geq & \sum_{i=1}^{N} \int_{Q} a_{i}(x, t, u, \nabla u(x, t)) \frac{\partial u}{\partial x_{i}} d x d t \\
& +\int_{Q} g(x, u, \nabla u) u(x, t) d x d t \\
\geq & \int_{Q}|\nabla u(x, t)|^{2} d x d t+\int_{Q}|u(x, t)|^{2} d x d t \\
= & \int_{0}^{T}\left(\int_{\Omega}\left(|\nabla u(x, t)|^{2}+|u(x, t)|^{2}\right) d x\right) d t \\
= & \int_{0}^{T}\|u(t)\|_{V}^{2} d t=\|u\|^{2}
\end{aligned}
$$

for all $u \in H$. Since the right side of the above inequality approaches $\infty$ as $\|u\| \rightarrow \infty$, for each $f \in L^{2}(Q)$ there exists $R=R(f)>0$ such that

$$
\langle L u+S u+C u-f, u\rangle>0
$$

for all $u \in D(L) \cap \partial B_{R}(0)$. By applying Theorem 12, we conclude that the equation $\mathrm{Lu}+\mathrm{Su}+\mathrm{Cu}=f$ is solvable in $D(L)$; that is, (82) admits at least one-weak solution.

In conclusion, we like to notice that the function $g$ depends on both $u$ and $\nabla u$, sublinear, and possibly nonmonotone with respect to $u$. Consequently, Theorem 15 improves those analogous results under monotonicity condition on $g$ with respect to $u$. Existence results in elliptic as well as parabolic problems under monotone nonlinearities independent of $\nabla u$; the reader is referred to $[7,9,17,20,21,25-28]$ and the references therein.

\section{Conflicts of Interest}

The author declares that there are no conflicts of interest regarding the publication of the paper.

\section{Acknowledgments}

The author is thankful to Virginia Tech for funding the article processing charge.

\section{References}

[1] F. E. Browder and P. Hess, "Nonlinear mappings of monotone type in Banach spaces," Journal of Functional Analysis, vol. 11, no. 3, pp. 251-294, 1972. 
[2] H. Brèzis, "Équations et inéquations non linéaires dans les espaces vectoriels en dualité," Annales de l'Institut Fourier, vol. 18, no. 1, pp. 115-175, 1968.

[3] T. M. Asfaw and A. G. Kartsatos, "A Browder topological degree theory for multi-valued pseudomonotone perturbations of maximal monotone operators," Advances in Mathematical Sciences and Applications, vol. 22, no. 1, pp. 91-148, 2012.

[4] T. M. Asfaw, "A new topological degree theory for pseudomonotone perturbations of the sum of two maximal monotone operators and applications," Journal of Mathematical Analysis and Applications, vol. 434, no. 1, Article ID 19800, pp. 967-1006, 2016.

[5] D. R. Adhikari and A. G. Kartsatos, "A new topological degree theory for perturbations of the sum of two maximal monotone operators," Nonlinear Analysis, vol. 74, no. 14, pp. 4622-4641, 2011.

[6] F. E. Browder, "Degree of mapping for nonlinear mappings of monotone type: densely defined mapping," Proceedings of the National Academy of Sciences of the United States of America, vol. 80, no. 8, pp. 2405-2407, 1983.

[7] S. C. Hu and N. S. Papageorgiou, "Generalizations of Browder's degree theory," Transactions of the American Mathematical Society, vol. 347, no. 1, pp. 233-259, 1995.

[8] J. Kobayashi and M. Otani, "Topological degree for (S)+mappings with maximal monotone perturbations and its applications to variational inequalities," Nonlinear Anal, vol. 59, pp. 147-172, 2004.

[9] T. M. Asfaw, "New variational inequality and surjectivity theories for perturbed noncoercive operators and application to nonlinear problems," Advances in Mathematical Sciences and Applications, vol. 24, no. 2, pp. 611-668, 2014.

[10] B. Ibrahimou and A. G. Kartsatos, "The Leray-Schauder approach to the degree theory for $(\mathrm{S}+)$-perturbations of maximal monotone operators in separable reflexive Banach spaces," Nonlinear Analysis, vol. 70, no. 12, pp. 4350-4368, 2009.

[11] V. Barbu, Nonlinear Differential Equations of Monotone Types in Banach Spaces, Springer Monographs in Mathematics, Springer, New York, NY, USA, 2010.

[12] D. Pascali and S. Sburlan, Nonlinear Mappings of Monotone Type, Sijthoff and Noordhoff, Bucharest, Romania, 1978.

[13] E. Zeidler, Nonlinear Functional Analysis and Its Applications, Springer-Verlag, New York, NY, USA, 1990.

[14] H. Brezis, M. G. Crandall, and A. Pazy, "Perturbations of nonlinear maximal monotone sets in Banach space," Communications on Pure and Applied Mathematics, vol. 23, pp. 123-144, 1970.

[15] A. G. Kartsatos, "New results in the perturbation theory of maximal monotone and $M$-accretive operators in Banach spaces," Transactions of the American Mathematical Society, vol. 348, no. 5, pp. 1663-1707, 1996.

[16] S. S. Zhang and Y. C. Chen, "Degree theory for multivalued (S) type mapping and fixed point theorems," Applied Mathematics and Mechanics, vol. 11, no. 5, pp. 409-421, 1990.

[17] N. Kenmochi, "Monotonicity and compactness methods for nonlinear variational inequalities," in Handbook of differential equations, pp. 203-298, Elsevier, North-Holland, Amsterdam, 2007.

[18] V. K. Le, "A range and existence theorem for pseudomonotone perturbations of maximal monotone operators," Proceedings of the American Mathematical Society, vol. 139, no. 5, pp. 16451658, 2011.
[19] T. M. Asfaw, "New surjectivity results for perturbed weakly coercive operators of monotone type in reflexive banach spaces," Nonlinear Analysis, vol. 113, pp. 209-229, 2015.

[20] V. Mustonen, "On elliptic operators in divergence form; old and new with applications," in Proceedings of the Function Spaces and Differential Operators and Nonl. Anal., pp. 188-200, 2004.

[21] F. E. Browder, "Pseudo-monotone operators and nonlinear elliptic boundary value problems on unbounded domains," Proceedings of the National Academy of Sciences of the United States of America, vol. 74, no. 7, pp. 2659-2661, 1977.

[22] J. Berkovits and V. Mustonen, “Topological degree for perturbations of linear maximal monotone mappings and applications to a class of parabolic problems," Rendiconti di Matematica 12, vol. 12, no. 3, pp. 597-621, 1992.

[23] R. Landes and V. Mustonen, "On pseudomonotone operators and nonlinear noncoercive variational problems on unbounded domains," Mathematische Annalen, vol. 248, no. 3, pp. 241-246, 1980.

[24] S. Carl, V. K. Le, and D. Motreanu, Nonsmooth Variational Problems and their Inequalities: Comparison Principles and Applications, Springer Monographs in Mathematics, Springer, New York, NY, USA, 2007.

[25] H. Brèzis and L. Nirenberg, "Characterizations of the ranges of some nonlinear operators and applications to boundary value problems," Annali Della Scuola Normale Superiore Di Pisa, vol. 5, no. 2, pp. 225-326, 1978.

[26] H. Brèzis and F. Browder, "Strongly nonlinear elliptic boundary value problems," Annali Della Scuola Normale Superiore Di Pisa, vol. 5, no. 3, pp. 587-603, 1978.

[27] S. Carl and V. K. Le, "Quasilinear parabolic variational inequalities with multi-valued lower-order terms," Zeitschrift für angewandte Mathematik und Physik, vol. 65, no. 5, pp. 845-864, 2014.

[28] P. Drabek, "The p-Laplacian-mascot of nonlinear analysis," Acta. Math.Univ.Comenianae 1, vol. 76, no. 1, pp. 85-98, 2007. 


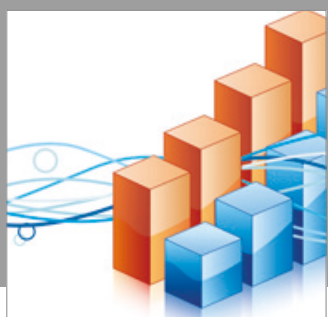

Advances in

Operations Research

vatersals

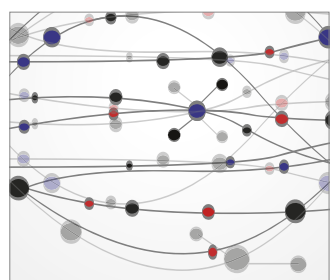

\section{The Scientific} World Journal
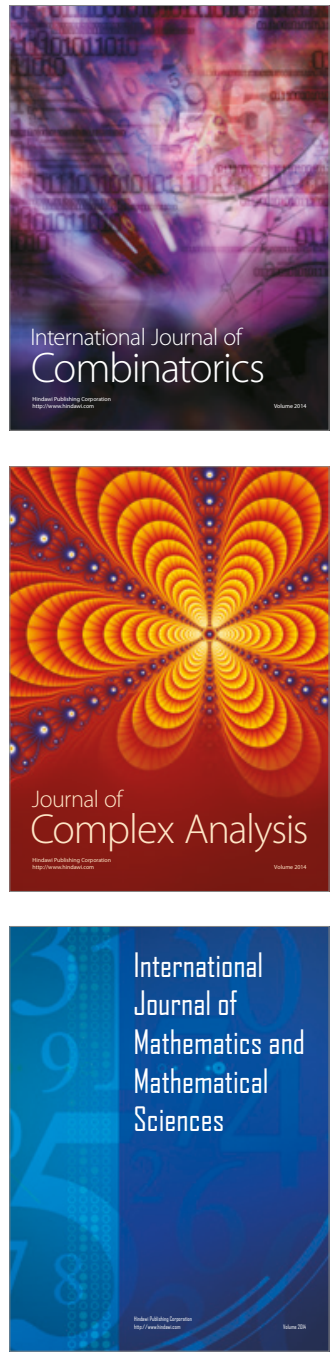
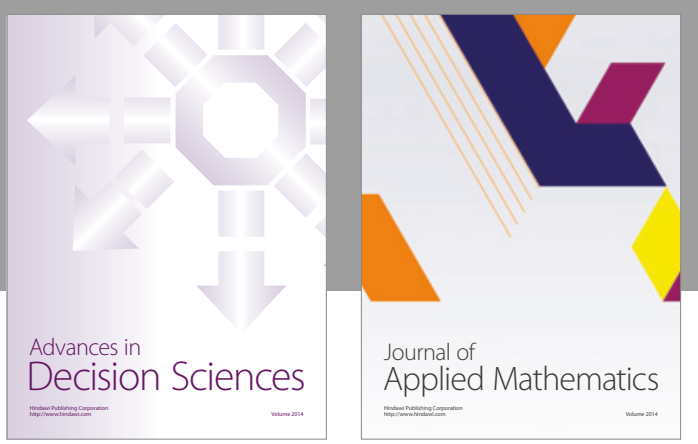

Algebra

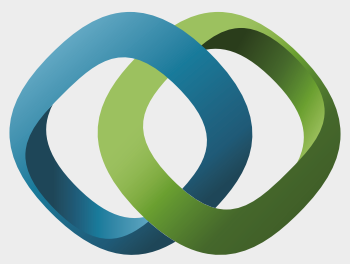

\section{Hindawi}

Submit your manuscripts at

https://www.hindawi.com
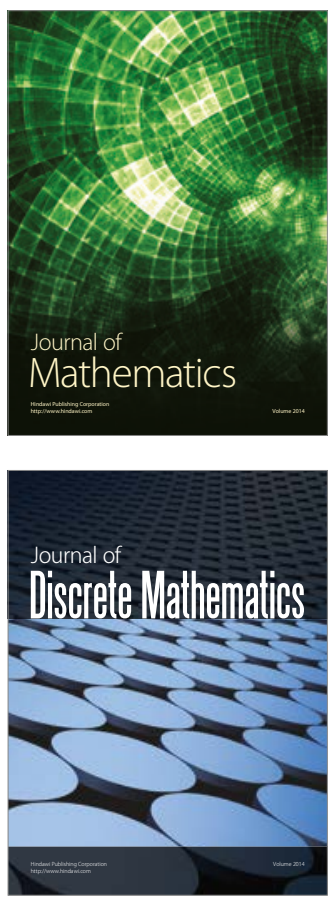

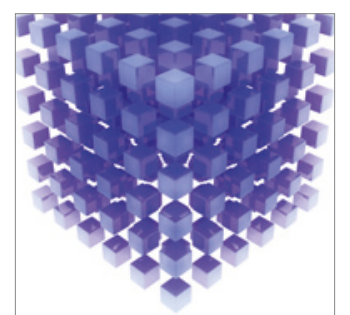

Mathematical Problems in Engineering
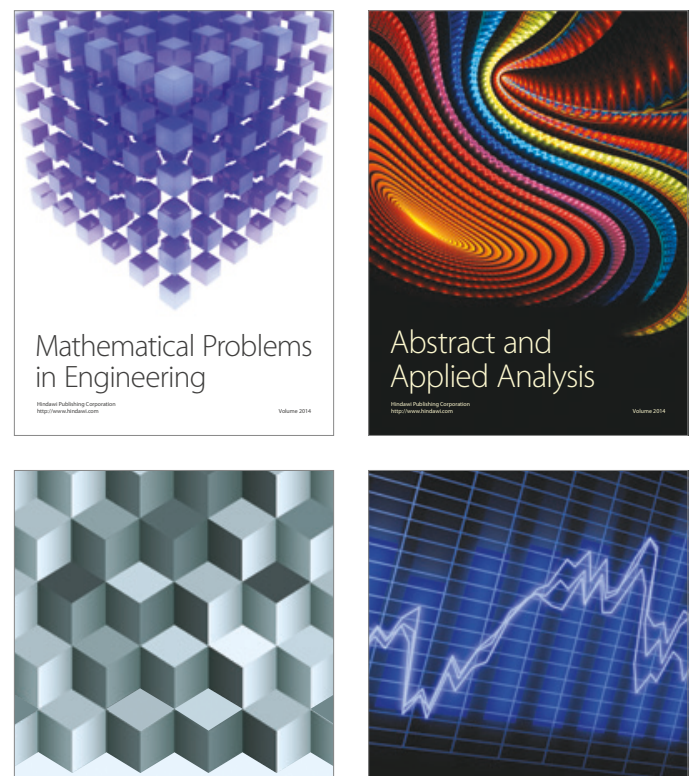

Journal of

Function Spaces

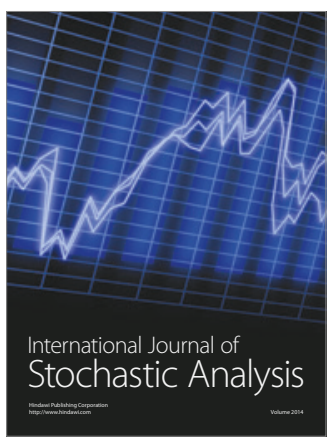

Probability and Statistics
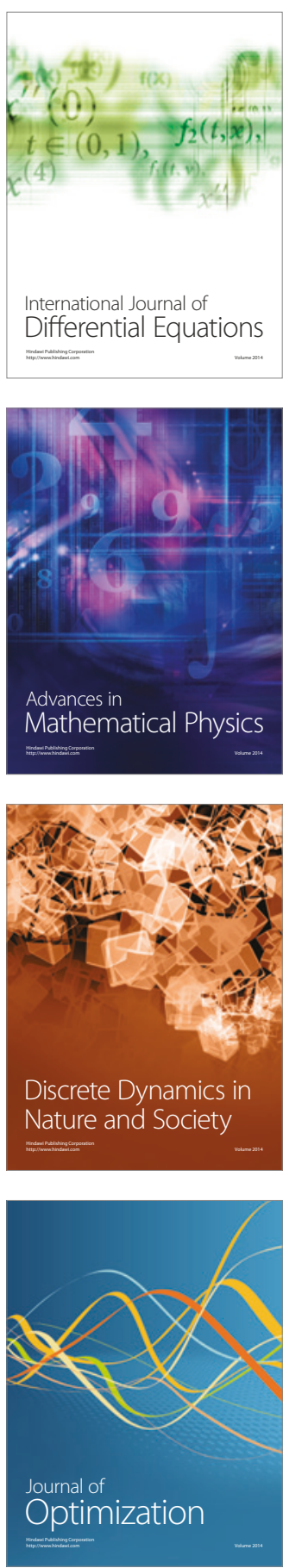